Review

\title{
Non-Invasive Biomarkers for Earlier Detection of Pancreatic Cancer-A Comprehensive Review
}

\author{
Greta Brezgyte $^{\dagger}$, Vinay Shah ${ }^{+}$, Daria Jach and Tatjana Crnogorac-Jurcevic * \\ Centre for Cancer Biomarkers and Biotherapeutics, Barts Cancer Institute, Queen Mary University of London, \\ London EC1M 6BQ, UK; g.brezgyte@qmul.ac.uk (G.B.); vinay.shah8@nhs.net (V.S.); d.jach@qmul.ac.uk (D.J.) \\ * Correspondence: t.c.jurcevic@qmul.ac.uk \\ + Shared first authorship.
}

Simple Summary: Pancreatic ductal adenocarcinoma (PDAC), which represents approximately $90 \%$ of all pancreatic cancers, is an extremely aggressive and lethal disease. It is considered a silent killer due to a largely asymptomatic course and late clinical presentation. Earlier detection of the disease would likely have a great impact on changing the currently poor survival figures for this malignancy. In this comprehensive review, we assessed over 4000 reports on non-invasive PDAC biomarkers in the last decade. Applying the Quality Assessment of Diagnostic Accuracy Studies (QUADAS-2) tool, we selected and reviewed in more detail 49 relevant studies reporting on the most promising candidate biomarkers. In addition, we also highlight the present challenges and complexities of translating novel biomarkers into clinical use.

check for updates

Citation: Brezgyte, G.; Shah, V.; Jach, D.; Crnogorac-Jurcevic, T.

Non-Invasive Biomarkers for Earlier Detection of Pancreatic Cancer-A Comprehensive Review. Cancers 2021, 13, 2722. https://doi.org/10.3390/ cancers 13112722

Academic Editor: Adam E. Frampton

Received: 22 April 2021

Accepted: 27 May 2021

Published: 31 May 2021

Publisher's Note: MDPI stays neutral with regard to jurisdictional claims in published maps and institutional affiliations.

Copyright: (C) 2021 by the authors. Licensee MDPI, Basel, Switzerland. This article is an open access article distributed under the terms and conditions of the Creative Commons Attribution (CC BY) license (https:/ / creativecommons.org/licenses/by/ $4.0 /)$.

\begin{abstract}
Pancreatic ductal adenocarcinoma (PDAC) carries a deadly diagnosis, due in large part to delayed presentation when the disease is already at an advanced stage. CA19-9 is currently the most commonly utilized biomarker for PDAC; however, it lacks the necessary accuracy to detect precursor lesions or stage I PDAC. Novel biomarkers that could detect this malignancy with improved sensitivity (SN) and specificity (SP) would likely result in more curative resections and more effective therapeutic interventions, changing thus the present dismal survival figures. The aim of this study was to systematically and comprehensively review the scientific literature on noninvasive biomarkers in biofluids such as blood, urine and saliva that were attempting earlier PDAC detection. The search performed covered a period of 10 years (January 2010_August 2020). Data were extracted using keywords search in the three databases: MEDLINE, Web of Science and Embase. The Quality Assessment of Diagnostic Accuracy Studies (QUADAS-2) tool was applied for study selection based on establishing the risk of bias and applicability concerns in Patient Selection, Index test (biomarker assay) and Reference Standard (standard-of-care diagnostic test). Out of initially over 4000 published reports, 49 relevant studies were selected and reviewed in more detail. In addition, we discuss the present challenges and complexities in the path of translating the discovered biomarkers into the clinical setting. Our systematic review highlighted several promising biomarkers that could, either alone or in combination with CA19-9, potentially improve earlier detection of PDAC. Overall, reviewed biomarker studies should aim to improve methodological and reporting quality, and novel candidate biomarkers should be investigated further in order to demonstrate their clinical usefulness. However, challenges and complexities in the path of translating the discovered biomarkers from the research laboratory to the clinical setting remain and would have to be addressed before a more realistic breakthrough in earlier detection of PDAC is achieved.
\end{abstract}

Keywords: pancreatic ductal adenocarcinoma; systematic review; biomarkers; QUADAS-2; early detection; non-invasive

\section{Introduction}

Pancreatic ductal adenocarcinoma (PDAC), which represents approximately $90 \%$ of all pancreatic cancers, is an extremely aggressive disease and one of the most lethal 
cancers. Its incidence almost equals its mortality, and it has a 5-year survival of only around 9\% [1,2]. It caused 7800 deaths in the United Kingdom (UK) in 2018 alone, and, according to GLOBOCAN, there were 458,918 new cases of pancreatic cancer and 432,242 pancreatic cancer-related deaths worldwide in the same year [3]. Furthermore, PDAC incidence is steadily increasing [4-7].

PDAC is considered a silent killer due to a largely asymptomatic course, late clinical presentation, and rapid progression [8], with fewer than $20 \%$ of PDAC patients being diagnosed at early, resectable stages I and II $[9,10]$. Importantly, if PDAC is detected when still a localized disease, the 5-year survival is around 32\% [11], but it can approach $70 \%$ following resection of incidentally diagnosed stage I tumors [12-14]. It is estimated that it takes at least 10 years between initiating mutation and the birth of parental founder [15]; combined with an increased PDAC incidence with age [5], resectable PDAC has likely already been growing for a number of years. We therefore further on use the term "earlier" rather than "early" stage.

To date, there exists no reliable, non-invasive screening test, either molecular or imaging-based, that will allow accurate PDAC detection at an earlier stage in asymptomatic patients. The conventional methods such as computerized tomography, magnetic resonance imaging or endoscopic ultrasonography are expensive and have low sensitivity (SN) and specificity (SP) for detection of small premalignant lesions [16]. Furthermore, the deep anatomic location of the pancreas makes detection of small-localized tumors unlikely during a routine abdominal examination [17].

In contrast, testing for biomarkers in body fluids such as serum, plasma, urine or saliva is minimally invasive, relatively inexpensive and could allow for earlier PDAC diagnosis [18]. The current gold-standard serum biomarker for PDAC is carbohydrate antigen 19-9 (CA19-9), also known as sialylated Lewis (a) antigen. It is a product of exocrine epithelial cells and various mucins secreted by PDAC cells that are normally found on the surface of erythrocytes [19]. The test was approved by the U.S. Food and Drug Administration (FDA) in 2002 [20]. However, it is recommended to be used only for disease monitoring and prognosis, since the performance of CA19-9 as a diagnostic biomarker is limited by poor SN, false-negative results in the individuals with Lewis negative phenotype and increased false positivity in several benign and malignant biliary disorders [21]. Furthermore, CA19-9 is not recommended as a screening marker due to its low positive predictive value $(0.5-0.9 \%)$ [22-24].

The accuracy of any tumor biomarkers assay, including CA19-9, is linked to the concept of threshold, or cut-off [25]. The CA19-9 cut-off values differ significantly between studies due to the lack of an international standard for CA19-9 and the difference in the assay design and instrument utilized [26]. When the two most frequently used assays for measuring CA19-9, Abbott Architect CA 19-9XR and Roche Elecsys CA19-9, were compared, despite being based on the same monoclonal antibody 1116-NS-19-9 [27], the Abbott assay measured significantly lower CA19-9 values than the Roche one in healthy and benign cases [28]. While the upper reference limit of $37 \mathrm{U} / \mathrm{mL}$, reaching mean SN and SP of $81 \%$ and $90 \%$, respectively, as first reported by Steinberg [29], is still able to sufficiently differentiate between controls and PDAC, the CA19-9 values obtained by different methods should not be used interchangeably, the method utilized should be clearly stated and the patients should be monitored using the same assay [28,30]. Thus, despite its role as the principle serological test for PDAC, the standardization of the laboratory testing and correct interpretation of high serum CA19-9 levels with the adequately established cut off has not yet been achieved [31]. The search for a new non-invasive PDAC biomarker with superior SN and SP to CA19-9, which will be easily identifiable and robustly measured in a fully standardized fashion in biofluids, is therefore being actively pursued.

Recent advances in molecular techniques have empowered the discovery of novel biomarkers for earlier detection of PDAC, resulting in a significant and constantly growing body of literature. Despite the discovery of thousands of biomarkers, relatively few have been adequately tested and validated, making the assessment of their potential 
challenging [32]. This sparked an EDRN (The Early Detection Research Network) workshop at National Cancer Institute (NCI) in 2016, where a selection of the most promising studies was reported [33]. A comprehensive update and critically appraised resource of information on potentially useful novel biomarkers for PDAC is, however, needed.

In the present study, we reviewed over 4000 articles and further assessed 49 published studies from the last decade describing biomarkers in serum, plasma, urine and saliva for earlier PDAC detection, in conjunction with serum CA 19-9, when available. We utilized the Quality Assessment of Diagnostic Accuracy Studies tool (QUADAS-2) to assess the quality of the selected studies and highlighted their potential clinical utility and limitations for earlier PDAC detection.

\section{Methods}

Two of the authors (G.B. and V.S.) independently conducted a systematic search of MEDLINE, Web of Science and Embase and assessed all the articles published between January 2010 and August 2020. A robust reproducible search strategy was created which utilized the MESH terms "Pancreas OR Pancreatic" AND "Ductal Adenocarcinoma OR PDAC OR Cancer OR Malignancy OR Adenocarcinoma OR Tumour OR Tumor" AND “Liquid Biopsy OR Biomarkers OR Screening OR Stool OR Markers OR Marker OR Urine OR Saliva OR Blood OR Non-invasive OR Non Invasive OR Fluid Biopsy OR Fluid Phase Biopsy OR Detection OR Diagnosis OR Diagnose OR Screen" to search for the potential biomarkers specific for this disease. The filters were adjusted to show the results for studies in the English language only. The article type was set to only include clinical and observational studies in Medline. In Medline and Embase, titles and abstracts were searched. In Web of Science, only titles were searched. PRISMA guidelines were used to assist in the structuring of this systematic review [34]. The inclusion and exclusion criteria for selecting the studies are listed in Table 1.

Table 1. Inclusion and Exclusion Criteria for study selection.

\begin{tabular}{c} 
Inclusion Criteria \\
\hline 1. Pancreatic Ductal Adenocarcinoma \\
2. Non-invasive method of obtaining a liquid biopsy e.g., plasma, serum, urine, saliva, stool \\
3. Original data with reported AUC, SN and SP of a proposed biomarker \\
4. Human studies \\
5. Manuscripts from January 2010 until August 2020 \\
\hline
\end{tabular}

\section{Exclusion Criteria}

1. No specification of what type of pancreatic cancer it was

2. Invasive procedures to obtain the biomarker e.g., tissue biopsy

3. No recorded data either of SN, SP and/or AUC for the tested biomarker

4. Biomarker used for a purpose other than detection e.g., prognostic biomarkers

5. Abstracts, Conference reports/writings, NHS reports, Review Articles

Abbreviations: AUC-Area Under the ROC (Receiver Operating Characteristic) Curve, SN-Sensitivity, SPSpecificity, NHS-National Health Service in UK.

Assessment of the methodological quality of reviewed articles was performed according to QUADAS-2, a validated quality assessment tool commonly used to assess the methodology of investigated studies, and as a measure to improve diagnostic accuracy [35]. With QUADAS-2, we were able to appraise three key domains, namely Patient Selection, Index Test and Reference Standard, for both risk of bias and applicability. For the Patients selection, we assessed if the sample groups were well described and if patient spectrum was representative of the disease. We also assessed if the selection of the controls was appropriate, i.e., if, in addition to healthy controls, samples collected from patients with benign pancreatic diseases at risk for progressing to PDAC were also included. Within the Applicability concerns, we assessed if the data for PDAC stages I/II were reported, as we were aiming to review biomarkers for earlier detection of PDAC. For the Index test (biomarker assay), we appraised how well the assay was described and executed and the 
results interpreted (SN/SP/AUC with 95\% confidence intervals (CIs)) and if the data were split into training and validation sets. To increase the validity of the results, QUADAS-2 also recommends that researchers do not know which study group each patient belongs to ("blinding") in order to reduce the effects of any foreknowledge in the interpretation of the biomarker assay results [36]. QUADAS-2 includes the need to specify a threshold value for the Index test prior to obtaining the results; however, this is not suitable for newly discovered biomarkers, where one of the aims is to determine the appropriate cut-offs with regards to the SN, SP and AUC. For the Reference Standard, we assessed if the study specifies that PDAC diagnosis was confirmed by histology and if the staging was pathological or clinical. However, as biomarker discovery studies are typically performed on retrospectively collected samples with an already established diagnosis, we additionally included a comparison of the new biomarker with CA19-9. Flow and Timing were excluded from our QUADAS-2 scoring, as it was difficult to assess this parameter, i.e., in the majority of the studies, it was unclear in what time frame were the diagnostic biopsy or imaging performed in respect to the sample collection and the biomarker testing.

\section{Results and Discussion}

A PRISMA flow diagram demonstrating the search strategy and study selection criteria that were applied is shown in Figure 1.

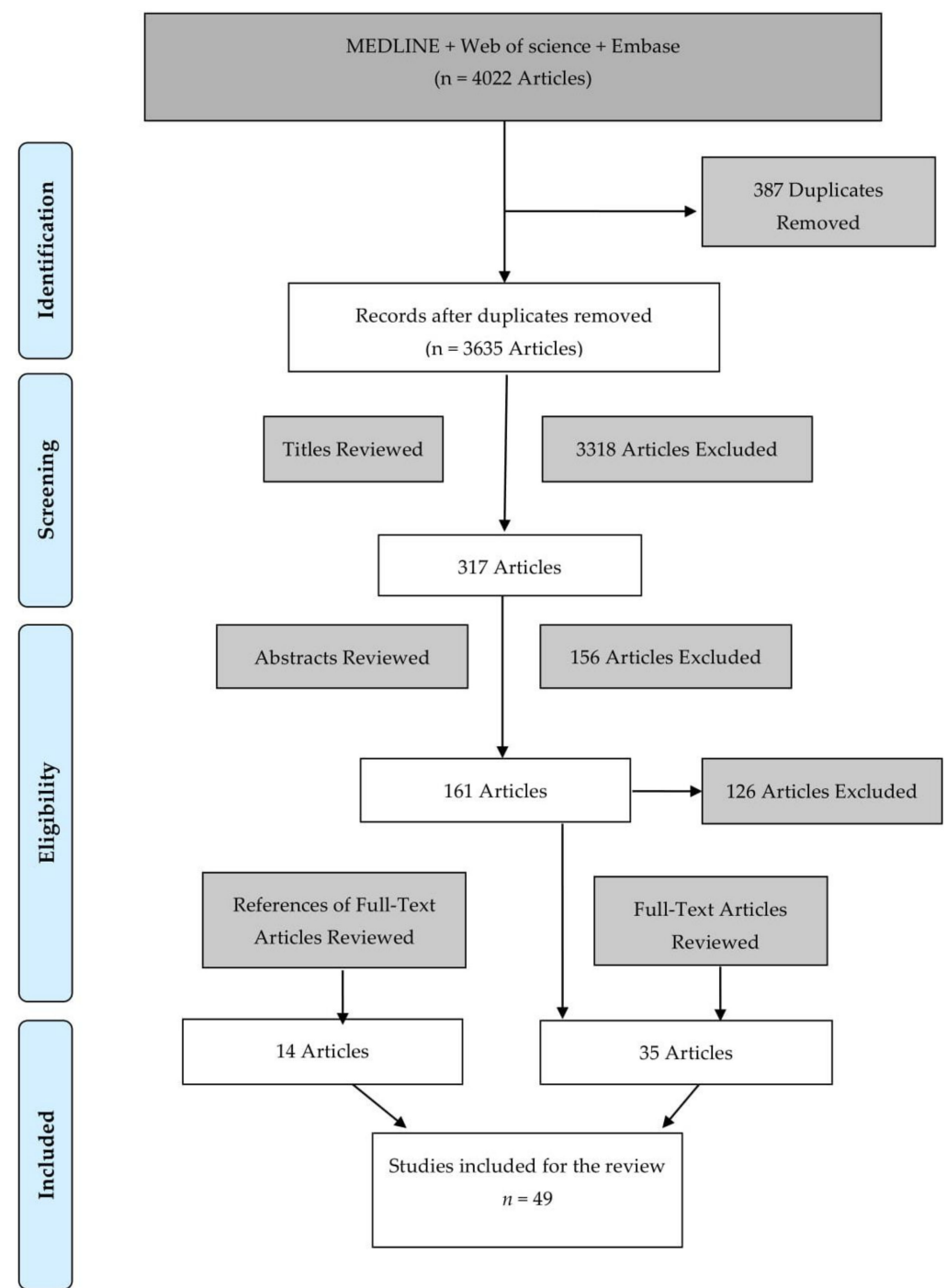

Figure 1. PRISMA Flow diagram of the search strategy and study selection. 
The overall quality of the 49 selected and reviewed studies is illustrated in Figure 2.

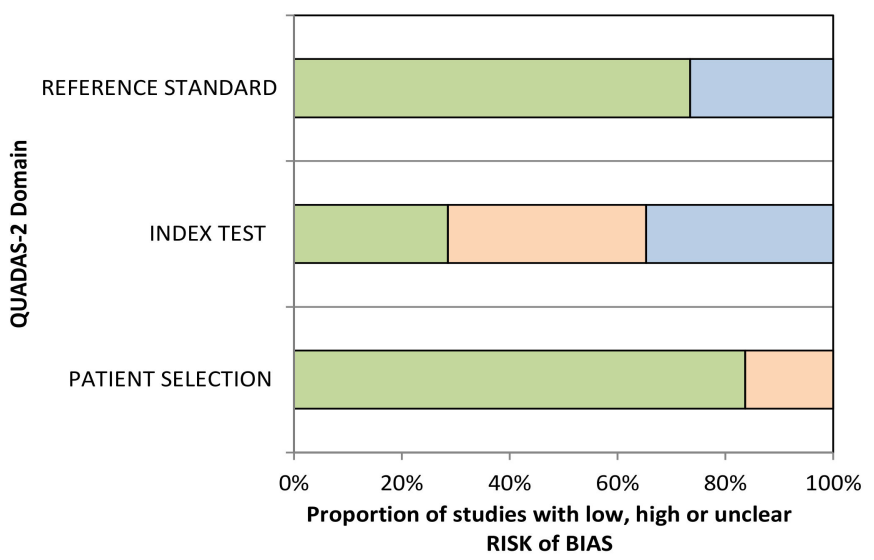

$\square$ Low $\square$ High $\square$ Unclear

Figure 2. Graphical representation of the overall quality of the selected studies.

In Figure 2 it is evident that the majority of the studies (70\%) had a low risk of bias in the Reference Standard domain, as the information on histological confirmation that samples originated from PDAC was specified. The unclear bias in this domain seen in approximately $30 \%$ of the studies was due to the absence of comparison of biomarkers with CA19-9. High risk of bias in the Index test (biomarker assay) was seen in $36 \%$ of studies and was associated with missing information on if the samples were analyzed in the blinded fashion. The lack of clarity, which was seen in the following $36 \%$ of the studies, was due to absence of robust statistical analysis of the data, i.e., splitting of the data into the training and the validation sets. Regarding patient selection criteria, around $20 \%$ of studies did not compare PDAC results to appropriate benign controls, leading to a high risk of bias. Around $20 \%$ of studies raised concerns about the applicability of the biomarkers for earlier PDAC detection, as they were not assessed in resectable stage I-II PDAC.

A graphical summary of the QUADAS-2 results with the descriptive score for each of the selected studies is presented in a tabular form in supplementary Appendix A, Table A1.

Table 2 summarizes the key findings from all 49 studies included in this review. 
Table 2. Key findings from included studies.

\begin{tabular}{|c|c|c|c|c|c|c|c|}
\hline Reference & $\begin{array}{l}\text { Specimen } \\
\text { Type }\end{array}$ & Biomarker & Clinical Setting & Subjects & Sensitivity (\%) & Specificity (\%) & AUC \\
\hline Gold et al., 2010 [37] & Serum & PAM4 & 68 PDAC, $19 \mathrm{HC}$ & PDAC vs. HC & 82.0 & 95.0 & $0.92(0.84-0.97)$ \\
\hline \multirow{3}{*}{$\begin{array}{l}\text { Joergensen et al., } \\
2010 \text { [38] }\end{array}$} & \multirow{3}{*}{ Serum } & CA19-9 & \multirow{3}{*}{$51 \mathrm{PDAC}, 52 \mathrm{HC}$} & \multirow{3}{*}{ PDAC vs. HC } & 86.0 & 73.0 & $0.84(0.77-0.92)$ \\
\hline & & MMP-9 & & & 58.8 & 34.6 & $0.50(0.39-0.61)$ \\
\hline & & TIMP1 & & & 47.1 & 69.2 & $0.64(0.53-0.74)$ \\
\hline \multirow{2}{*}{$\begin{array}{l}\text { Marten et al., } \\
2010 \text { [39] }\end{array}$} & \multirow{2}{*}{ Plasma } & $\mathrm{siC} 3 \mathrm{~b}$ & 157 PDAC, 38 HC & $\begin{array}{c}2-4 \text { mo prior } \\
\text { radiologically } \\
\text { defined recurrence }\end{array}$ & 54.0 & 94.0 & 0.85 \\
\hline & & $\mathrm{siC} 3 \mathrm{~b}$ & & $\begin{array}{c}0-2 \text { mo prior } \\
\text { radiologically } \\
\text { defined recurrence }\end{array}$ & 62.0 & 94.0 & 0.84 \\
\hline \multirow{2}{*}{ Brand et al., 2011 [17] } & \multirow{2}{*}{ Serum } & $\begin{array}{c}\text { CA19-9 } \\
\text { CA19-9 + ICAM-1 + OPG } \\
\text { CA19-9 + CEA + TIMP-1 }\end{array}$ & $\begin{array}{l}160 \mathrm{PDAC} \\
74 \mathrm{BPD} \\
107 \mathrm{HC}\end{array}$ & $\begin{array}{c}\text { TS: } \\
\text { PDAC vs. HC } \\
\text { PDAC vs. BPD } \\
\text { PDAC vs. HC }\end{array}$ & $\begin{array}{l}57,2 \\
88.0 \\
76.0\end{array}$ & $\begin{array}{l}90.0 \\
90.0 \\
90.0\end{array}$ & $\begin{array}{c}0.83(0.81-0.86) \\
0.93(0.91-0.95) \\
0.86\end{array}$ \\
\hline & & $\begin{array}{c}\text { CA19-9 } \\
\text { CA19-9 + ICAM-1 + OPG } \\
\text { CA19-9 + CEA + TIMP-1 } \\
\text { CA19-9 }\end{array}$ & $\begin{array}{l}173 \mathrm{PDAC} \\
70 \mathrm{BPD} \\
120 \mathrm{HC}\end{array}$ & $\begin{array}{c}\text { VS: } \\
\text { PDAC vs. BPD } \\
\text { PDAC vs. HC } \\
\text { PDAC vs. BPC } \\
\text { PDAC vs. BPC }\end{array}$ & $\begin{array}{l}56.4 \\
78.0 \\
71.2 \\
52.1\end{array}$ & $\begin{array}{l}90.0 \\
94.1 \\
88.6 \\
90.2\end{array}$ & $\begin{array}{l}0.82(0.78-0.86) \\
0.91(0.88-0.95) \\
0.83(0.88-0.89) \\
0.78(0.74-0.83)\end{array}$ \\
\hline \multirow{8}{*}{ Park et al., 2012 [21] } & \multirow{8}{*}{ Serum } & Cathepsin D & & & 54.0 & 80.0 & 0.67 \\
\hline & & MMP-7 & 109 PDAC, & TS: PDAC vs. HC + & 72.0 & 80.0 & 0.81 \\
\hline & & CA19-9 & $40 \mathrm{HC}, 30 \mathrm{CP}$ & $\mathrm{CP}$ & 74.0 & 80.0 & 0.84 \\
\hline & & CA19-9 + Cathepsin D + MMP-7 & & & 88.0 & 80.0 & $0.90(\mathrm{p}=0.002)$ \\
\hline & & Cathepsin D & & & 53.0 & 79.0 & 0.65 \\
\hline & & MMP-7 & 129 PDAC, & VS: PDAC vs. HC + & 65.0 & 79.0 & 0.77 \\
\hline & & CA19-9 & $74 \mathrm{HC}, 72 \mathrm{CP}$ & $\mathrm{CP}$ & 78.0 & 84.0 & 0.88 \\
\hline & & CA19-9 + Cathepsin D + MMP-7 & & & 89.0 & 77.0 & $0.91(\mathrm{p}=0.002)$ \\
\hline \multirow{2}{*}{$\begin{array}{l}\text { Capello et al., } \\
2013 \text { [40] }\end{array}$} & \multirow{2}{*}{ Serum } & \multirow{2}{*}{ EZR-autoantibody } & \multirow{2}{*}{$\begin{array}{l}69 \text { PDAC, } 46 \text { CP, } 60 \mathrm{HC} \text {, } \\
12 \text { Aim, } 50 \text { Non-PDAC }\end{array}$} & $\begin{array}{l}\text { PDAC vs. } \mathrm{HC}+\mathrm{CP}+ \\
\text { Aim }\end{array}$ & 93.2 & 75.5 & 0.90 \\
\hline & & & & $\begin{array}{l}\text { PDAC vs. non-PDAC } \\
\text { cancer }\end{array}$ & 94.9 & 96.4 & 0.99 \\
\hline
\end{tabular}


Table 2. Cont.

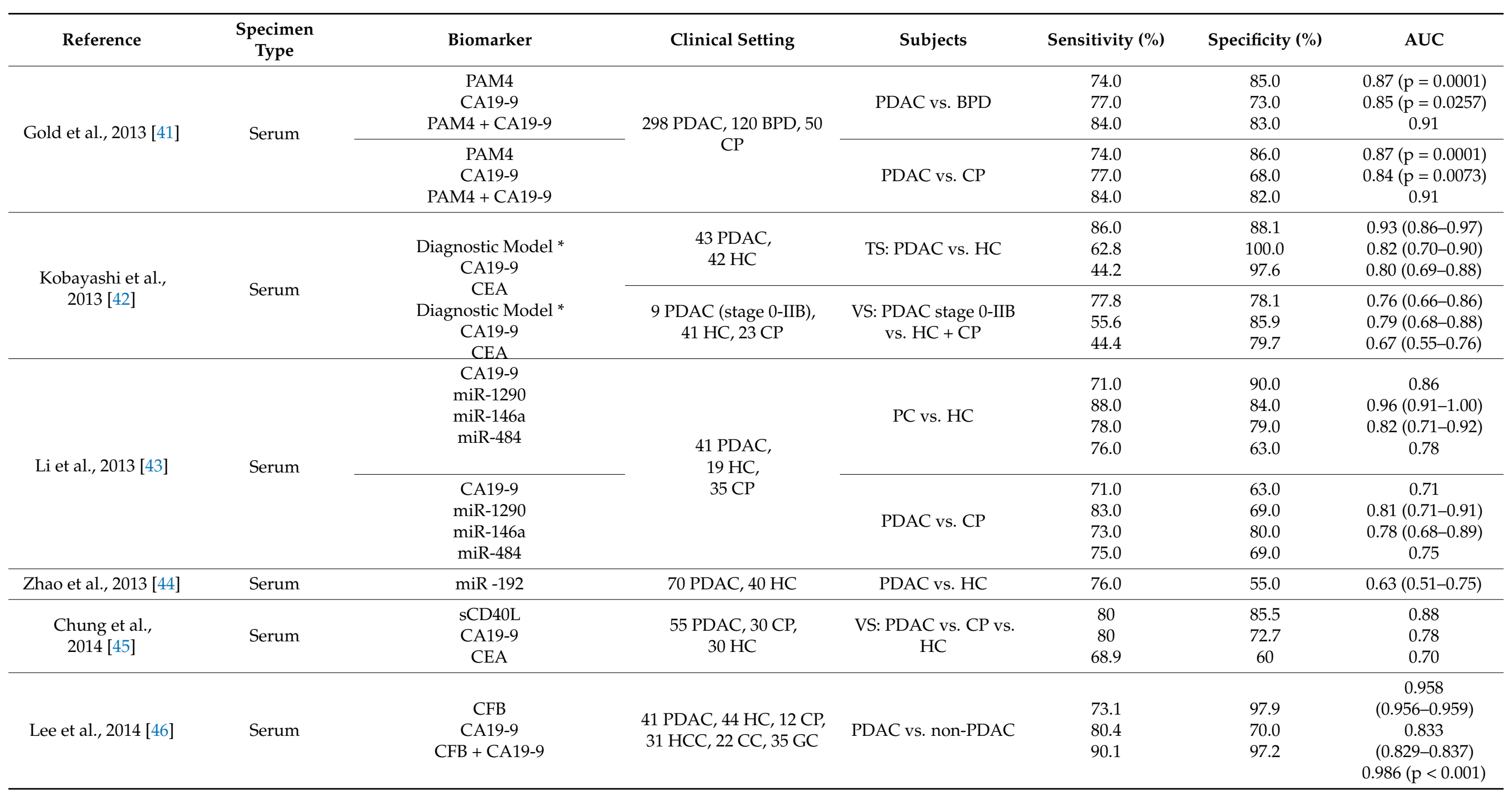


Table 2. Cont

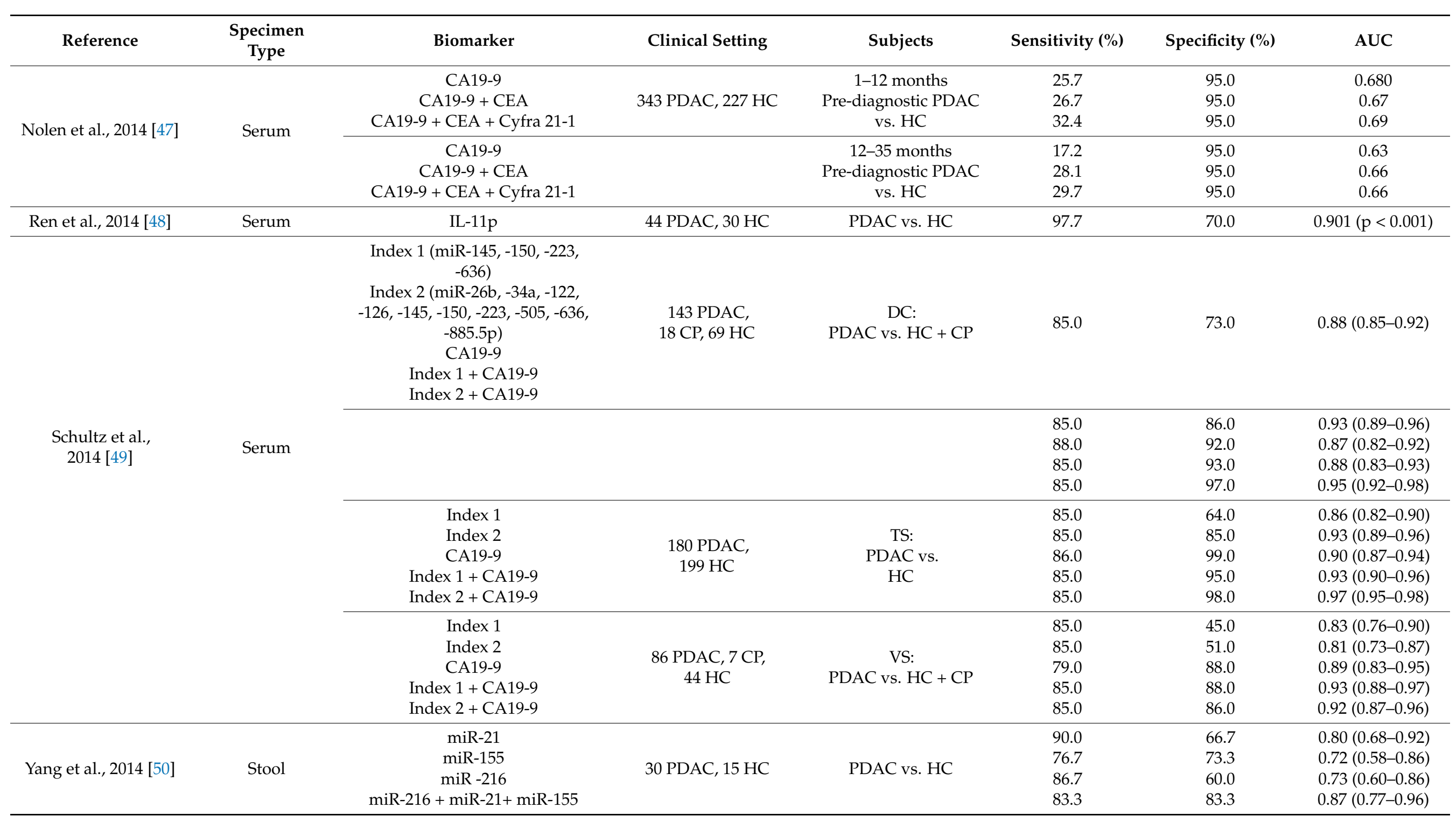


Table 2. Cont.

\begin{tabular}{|c|c|c|c|c|c|c|c|}
\hline Reference & $\begin{array}{l}\text { Specimen } \\
\text { Type }\end{array}$ & Biomarker & Clinical Setting & Subjects & Sensitivity (\%) & Specificity (\%) & AUC \\
\hline \multirow{2}{*}{$\begin{array}{l}\text { Zhang et al., } \\
2014 \text { [51] }\end{array}$} & \multirow{2}{*}{ Serum } & $\begin{array}{l}\text { CA19-9 + Albumin + CRP + IL-8 } \\
\text { CA19-9 } \\
\text { CA19-9 + Albumin + CRP + IL-8 } \\
\text { CA19-9 }\end{array}$ & $\begin{array}{l}163 \text { PDAC (77 Early } \\
\text { stage I-II), } \\
109 \mathrm{BC}, \\
200 \mathrm{HC}\end{array}$ & $\begin{array}{c}\text { All stage PDAC vs. } \\
\text { HC } \\
\text { All stage PDAC vs. } \\
\text { HC } \\
\text { Early stage PDAC vs. } \\
\text { HC } \\
\text { Early stage PDAC vs. } \\
\text { HC }\end{array}$ & $\begin{array}{l}99.4 \\
80.6 \\
96.1 \\
72.7\end{array}$ & $\begin{array}{l}90.0 \\
90.0 \\
90.0 \\
90.0\end{array}$ & $\begin{array}{l}0.98(0.97-1.00) \\
0.85(0.80-0.90) \\
0.97(0.95-1.00) \\
0.83(0.75-0.90)\end{array}$ \\
\hline & & $\begin{array}{c}\text { CA19-9 + } \mathrm{CO} 2+\mathrm{CRP}+\mathrm{IL}-6 \\
\text { CA19-9 } \\
\mathrm{CA} 19-9+\mathrm{CO} 2+\mathrm{CRP}+\mathrm{IL}-6 \\
\text { CA19-9 }\end{array}$ & & $\begin{array}{c}\text { All stage PDAC vs. } \\
\text { BC } \\
\text { All stage PDAC vs. } \\
\text { BC } \\
\text { Early stage PDAC vs. } \\
\text { BC } \\
\text { Early stage PDAC vs. } \\
\text { BC }\end{array}$ & $\begin{array}{l}74.2 \\
53.4 \\
75.3 \\
40.3\end{array}$ & $\begin{array}{l}90.0 \\
90.0 \\
90.0 \\
90.0\end{array}$ & $\begin{array}{l}0.89(0.86-0.93) \\
0.75(0.69-0.81) \\
0.87(0.82-0.93) \\
0.69(0.61-0.78)\end{array}$ \\
\hline \multirow{4}{*}{ Han et al., 2015 [53] } & \multirow{4}{*}{ Serum } & $\begin{array}{c}\text { Dickkopf-1 (DKK1) } \\
\text { CA19-9 }\end{array}$ & $\begin{array}{c}140 \text { PDAC, (62 Early } \\
\text { stage I-II), } \\
48 \mathrm{HC}, \\
18 \mathrm{BPT}, \\
26 \mathrm{CP}\end{array}$ & $\begin{array}{c}\text { PDAC vs. HC }+\mathrm{BPT} \\
+\mathrm{CP}\end{array}$ & $\begin{array}{l}89.3 \\
73.6\end{array}$ & $\begin{array}{l}79.4 \\
83.7\end{array}$ & $\begin{array}{l}0.92(0.88-0.95) \\
0.85(0.80-0.90)\end{array}$ \\
\hline & & $\begin{array}{c}\text { Dickkopf-1 (DKK1) } \\
\text { CA19-9 }\end{array}$ & & PDAC vs. $\mathrm{BPT}+\mathrm{CP}$ & $\begin{array}{l}89.3 \\
73.6\end{array}$ & $\begin{array}{l}72.7 \\
81.8\end{array}$ & $\begin{array}{l}0.89(0.83-0.95) \\
0.83(0.77-0.89)\end{array}$ \\
\hline & & $\begin{array}{c}\text { Dickkopf-1 (DKK1) } \\
\text { CA19-9 }\end{array}$ & & $\begin{array}{c}\text { Early-PDAC vs. HC + } \\
\text { BPT + CP }\end{array}$ & $\begin{array}{l}85.5 \\
64.5\end{array}$ & $\begin{array}{l}79.3 \\
83.7\end{array}$ & $\begin{array}{l}0.89(0.84-0.94) \\
0.81(0.74-0.89)\end{array}$ \\
\hline & & $\begin{array}{c}\text { Dickkopf-1 (DKK1) } \\
\text { CA19-9 }\end{array}$ & & $\begin{array}{c}\text { Early-PDAC vs. BPT } \\
+\mathrm{CP}\end{array}$ & $\begin{array}{l}85.5 \\
64.5\end{array}$ & $\begin{array}{l}72.7 \\
81.8\end{array}$ & $\begin{array}{l}0.85(0.78-0.93) \\
0.78(0.70-0.87)\end{array}$ \\
\hline
\end{tabular}


Table 2. Cont.

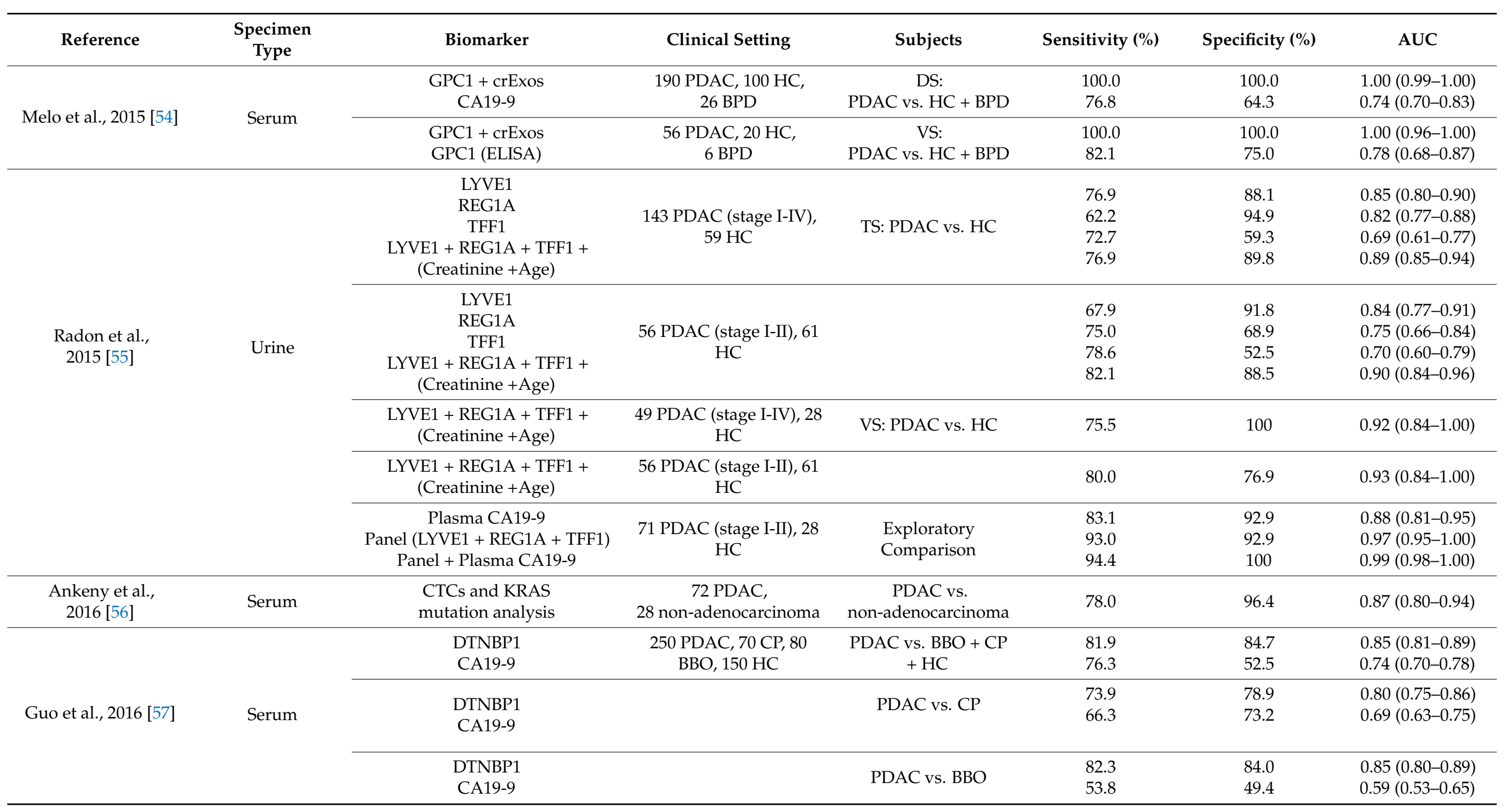


Table 2. Cont.

\begin{tabular}{|c|c|c|c|c|c|c|c|}
\hline Reference & $\begin{array}{l}\text { Specimen } \\
\text { Type }\end{array}$ & Biomarker & Clinical Setting & Subjects & Sensitivity (\%) & Specificity (\%) & AUC \\
\hline $\begin{array}{l}\text { Henriksen et al., } \\
\quad 2016[58]\end{array}$ & Plasma & $\begin{array}{c}\text { (Model13): age }>65+\text { BMP3+ RASSF1A+ BNC1+ } \\
\text { MESTv2+ TFPI2+ APC+ SFRP1 + } \\
\text { SFRP2 }\end{array}$ & $\begin{array}{l}95 \text { PDAC, } 97 \text { CP, } \\
27 \text { "screened negative" }\end{array}$ & $\begin{array}{l}\text { PDAC vs. screened } \\
\text { negative }+\mathrm{CP}\end{array}$ & 73.0 & 83.0 & $0.86(0.81-0.91)$ \\
\hline $\begin{array}{l}\text { Sogawa et al., } \\
2016[59]\end{array}$ & Serum & $\begin{array}{l}\text { C4BPA } \\
\text { CA19-9 } \\
\text { CEA }\end{array}$ & $\begin{array}{l}18 \text { PDAC (stage I-II), } \\
40 \mathrm{HC}, \\
20 \mathrm{CP}\end{array}$ & $\begin{array}{l}\text { PDAC stage I-II vs. HC } \\
+\mathrm{CP}\end{array}$ & $\begin{array}{l}50.0 \\
22.2 \\
22.2\end{array}$ & $\begin{array}{l}95.4 \\
95.4 \\
95.4\end{array}$ & $\begin{array}{c}0.91(\mathrm{p}<0.001) \\
0.74 \\
0.87\end{array}$ \\
\hline $\begin{array}{l}\text { Yoneyama et al., } \\
2016 \text { [60] }\end{array}$ & Serum & $\begin{array}{l}\text { CA19-9 } \\
\text { IGFBP2 } \\
\text { IGFBP3 }\end{array}$ & $\begin{array}{l}38 \text { PDAC (stage I-II), } \\
65 \text { HC }\end{array}$ & Stage I-II PDAC vs. HC & $\begin{array}{l}60.5 \\
68.4 \\
76.3\end{array}$ & $\begin{array}{l}92.3 \\
67.7 \\
70.7\end{array}$ & $\begin{array}{l}0.84(0.75-0.93) \\
0.71(0.60-0.81) \\
0.77(0.67-0.86)\end{array}$ \\
\hline \multirow{3}{*}{$\begin{array}{l}\text { Balasenthil } \\
\text { et al., } 2017 \text { [61] }\end{array}$} & \multirow{3}{*}{ Plasma } & CA19-9 & $\begin{array}{c}55 \text { PDAC (stage IA/ IB-IIA), } \\
61 \mathrm{HC}\end{array}$ & Stage IA/ IB-IIA vs. HC & 71.0 & 61.0 & $0.74(0.64-0.84)$ \\
\hline & & TNC + TFP1 + CA19-9 & $\begin{array}{l}55 \text { PDAC (stage IA/ IB-IIA), } \\
62 \mathrm{CP}\end{array}$ & Stage IA/ IB-IIA vs. CP & 73.0 & 82.0 & $0.79(0.70-0.87)$ \\
\hline & & $\begin{array}{c}\text { CA19-9 } \\
\text { TNC + TFP1 + CA19-9 }\end{array}$ & & & $\begin{array}{l}71.0 \\
73.0\end{array}$ & $\begin{array}{l}44.0 \\
71.0\end{array}$ & $\begin{array}{l}0.69(0.58-0.79) \\
0.75(0.65-0.84)\end{array}$ \\
\hline \multirow{2}{*}{$\begin{array}{l}\text { Yang et al., } \\
2017 \text { [62] }\end{array}$} & \multirow[t]{2}{*}{ Plasma } & $\begin{array}{c}\text { EGFR } \\
\text { EPCAM } \\
\text { HER2 } \\
\text { MUC1 } \\
\text { GPC1 } \\
\text { WNT2 } \\
\text { GRP94 } \\
\text { B7-H3 } \\
\text { EGFR + EPCAM + HER2 + MUC1 } \\
\text { EGFR + EPCAM + GPC1 + WNT2 } \\
\text { EGFR + EPCAM + MUC1 + GPC1 + WNT2 } \\
\text { EGFR + EPCAM + HER2 + MUC1 + GPC1 + } \\
\text { WNT2 }\end{array}$ & $22 \mathrm{PDAC}, 10 \mathrm{HC}$ & $\begin{array}{c}\text { TS: } \\
\text { PDAC vs. HC }\end{array}$ & $\begin{array}{c}73 \\
73 \\
59 \\
36 \\
55 \\
77 \\
73 \\
50 \\
91 \\
100 \\
100 \\
100\end{array}$ & $\begin{array}{c}100 \\
100 \\
90 \\
100 \\
60 \\
90 \\
70 \\
100 \\
100 \\
100 \\
100 \\
100\end{array}$ & $\begin{array}{l}0.90(0.79-1) \\
0.88(0.77-0.99) \\
0.72(0.55-0.89) \\
0.66(0.48-0.84) \\
0.48(0.28-0.67) \\
0.84(0.71-0.96) \\
0.73(0.55-0.90) \\
0.75(0.58-0.93) \\
0.99(0.97-1) \\
1.0 \\
1.0 \\
1.0\end{array}$ \\
\hline & & $\begin{array}{c}\text { EGFR } \\
\text { EPCAM } \\
\text { HER2 } \\
\text { MUC1 } \\
\text { GPC1 } \\
\text { WNT2 } \\
\text { GRP94 } \\
\text { EGFR + EPCAM + HER2 + MUC1 } \\
\text { EGFR + EPCAM + GPC1 + WNT2 } \\
\text { EGFR + EPCAM + MUC1 + GPC1 + WNT2 } \\
\text { EGFR + EPCAM + HER2 + MUC1 + GPC1 + } \\
\text { WNT2 }\end{array}$ & $\begin{array}{l}22 \text { PDAC, } 8 \text { CP, } 5 \text { BPD, } \\
8 \text { other abdominal } \\
\text { indications }\end{array}$ & $\begin{array}{c}\text { VS: } \\
\text { PDAC vs. CP vs. BPD } \\
\text { vs. other abdominal } \\
\text { indications }\end{array}$ & $\begin{array}{l}59 \\
45 \\
59 \\
36 \\
82 \\
64 \\
55 \\
86 \\
82 \\
86 \\
95\end{array}$ & $\begin{array}{l}76 \\
95 \\
85 \\
90 \\
52 \\
76 \\
71 \\
86 \\
90 \\
81 \\
81\end{array}$ & $\begin{array}{l}67(51-81) \\
70(54-83) \\
72(56-85) \\
63(47-77) \\
67(51-81) \\
70(54-83) \\
63(47-77) \\
86(72-95) \\
86(72-95) \\
84(69-93) \\
88(75-96)\end{array}$ \\
\hline
\end{tabular}


Table 2. Cont.

\begin{tabular}{|c|c|c|c|c|c|c|c|}
\hline Reference & $\begin{array}{l}\text { Specimen } \\
\text { Type }\end{array}$ & Biomarker & Clinical Setting & Subjects & Sensitivity (\%) & Specificity (\%) & AUC \\
\hline \multirow[t]{2}{*}{ Capello et al., 2017 [63] } & \multirow[t]{2}{*}{$\begin{array}{l}\text { Serum/ } \\
\text { Plasma }\end{array}$} & $\begin{array}{c}\text { CA19-9 } \\
\text { TIMP1 + LRG1 + CA19-9 } \\
\text { TIMP1 + LRG1 + CA19-9 (“OR" } \\
\text { Rule) } \\
\text { CA19-9 }\end{array}$ & $\begin{array}{l}39 \text { early stage PDAC, } \\
82 \mathrm{HC}\end{array}$ & $\begin{array}{l}\text { TS: early stage PDAC } \\
\text { vs. HC }\end{array}$ & $\begin{array}{l}53.8 \\
66.7 \\
72.6 \\
84.9\end{array}$ & $\begin{array}{l}95.0 \\
95.0 \\
95.0 \\
95.0\end{array}$ & $\begin{array}{l}0.82(0.74-0.91) \\
0.89(0.82-0.96) \\
0.88(0.81-0.96) \\
0.95(0.92-0.98)\end{array}$ \\
\hline & & $\begin{array}{c}\text { CA19-9 } \\
\text { TIMP1 + LRG1 + CA19-9 }\end{array}$ & $\begin{array}{l}73 \text { early stage PDAC, } \\
60 \mathrm{HC}\end{array}$ & $\begin{array}{l}\text { VS: early stage PDAC } \\
\text { vs. HC }\end{array}$ & $\begin{array}{l}84.9 \\
28.8\end{array}$ & $\begin{array}{l}95.0 \\
95.0\end{array}$ & $\begin{array}{l}0.96(0.89-1.00) \\
0.82(0.74-0.91)\end{array}$ \\
\hline Hussein et al., 2017 [64] & Serum & $\begin{array}{c}\text { miR-22-3p } \\
\text { miR-642b-3p } \\
\text { miR-885-5p } \\
\text { CA19-9 }\end{array}$ & $\begin{array}{c}35 \text { PDAC (33 early stage, } 2 \\
\text { late stage) } \\
15 \mathrm{HC}\end{array}$ & PDAC vs. HC & $\begin{array}{l}97.1 \\
100 \\
100 \\
91.4\end{array}$ & $\begin{array}{l}93.3 \\
100 \\
100 \\
100\end{array}$ & $\begin{array}{l}0.94(\mathrm{p}<0.001) \\
1.00(\mathrm{p}<0.001) \\
1.00(\mathrm{p}<0.001) \\
0.92(\mathrm{p}<0.001)\end{array}$ \\
\hline \multirow{4}{*}{ Kaur et al., 2017 [65] } & \multirow{4}{*}{ Serum } & $\begin{array}{l}\text { MUC5AC } \\
\text { CA19-9 }\end{array}$ & $\begin{array}{c}70 \text { PDAC (stage I or II), } \\
43 \mathrm{CP}, \\
35 \mathrm{HC} \\
30 \mathrm{BC}\end{array}$ & Early PDAC vs. HC & $\begin{array}{l}83.0 \\
56.0\end{array}$ & $\begin{array}{l}80.0 \\
95.0\end{array}$ & $\begin{array}{l}0.87(0.79-0.95) \\
0.72(0.59-0.84)\end{array}$ \\
\hline & & $\begin{array}{c}\text { MUC5AC } \\
\text { CA19-9 }\end{array}$ & & Early PDAC vs. BC & $\begin{array}{l}67.0 \\
48.0\end{array}$ & $\begin{array}{l}87.0 \\
89.0\end{array}$ & $\begin{array}{l}0.85(0.76-0.93) \\
0.71(0.59-0.83)\end{array}$ \\
\hline & & $\begin{array}{l}\text { MUC5AC } \\
\text { CA19-9 }\end{array}$ & & Early PDAC vs. CP & $\begin{array}{l}83.0 \\
48.0\end{array}$ & $\begin{array}{l}77.0 \\
86.0\end{array}$ & $\begin{array}{l}0.84(0.76-0.92) \\
0.62(0.50-0.74)\end{array}$ \\
\hline & & $\begin{array}{c}\text { MUC5AC } \\
\text { CA19-9 } \\
\text { MUC5AC + CA19-9 }\end{array}$ & & $\underset{\mathrm{CP}}{\text { PDAC vs. } \mathrm{HC}+\mathrm{BC}+}$ & $\begin{array}{l}89.0 \\
79.0 \\
83.0\end{array}$ & $\begin{array}{l}70.0 \\
43.0 \\
83.0\end{array}$ & $\begin{array}{l}0.88(0.83-0.93) \\
0.61(0.54-0.68) \\
0.91(0.86-0.95)\end{array}$ \\
\hline \multirow{2}{*}{ Kim et al., 2017 [66] } & \multirow{2}{*}{ Plasma } & $\begin{array}{c}\text { CA19-9 }(\geq 55) \\
\text { THBS2 }(36 \text { ng } / \text { mL cut-off }) \\
\text { CA19-9 + THBS2 }\end{array}$ & $\begin{array}{c}58 \text { (stage I-II, phase 2a), } \\
80 \mathrm{HC}\end{array}$ & $\begin{array}{l}\text { PDAC stage I or II vs. } \\
\text { HC }\end{array}$ & $\begin{array}{l}69.0 \\
33.0 \\
74.1\end{array}$ & $\begin{array}{l}100 \\
96.0 \\
96.3\end{array}$ & $\begin{array}{l}0.85(0.80-0.89) \\
0.83(0.78-0.89) \\
0.95(0.92-0.98)\end{array}$ \\
\hline & & $\begin{array}{c}\text { CA19-9 } \\
\text { THBS2 } \\
\text { CA19-9 + THBS2 } \\
\end{array}$ & $\begin{array}{c}88 \text { (stage I-II, phase 2b), } \\
140 \text { HC }\end{array}$ & & $\begin{array}{l}77.7 \\
58.4 \\
88.3\end{array}$ & $\begin{array}{l}98.6 \\
93.6 \\
92.9\end{array}$ & $\begin{array}{l}0.83(0.79-0.97) \\
0.89(0.85-0.92) \\
0.96(0.94-0.98)\end{array}$ \\
\hline Lai et al., 2017 [67] & Plasma & $\begin{array}{c}\text { CA19-9 } \\
\text { miR-10b } \\
\text { miR-21 } \\
\text { miR-30c } \\
\text { miR-106b } \\
\text { miR-20a } \\
\text { miR-181a } \\
\text { miR-483 } \\
\text { miR-let7a } \\
\text { miR-122 }\end{array}$ & 29 PDAC, 6 HC & PDAC vs. HC & $\begin{array}{c}86.0 \\
100.0 \\
86.0 \\
100.0 \\
97.0 \\
93.0 \\
97.0 \\
66.0 \\
93.0 \\
100.0\end{array}$ & $\begin{array}{c}100.0 \\
100.0 \\
100.0 \\
100.0 \\
100.0 \\
100.0 \\
100.0 \\
67.0 \\
100.0 \\
67.0\end{array}$ & $\begin{array}{l}0.92(\mathrm{p}<0.001) \\
1.00(\mathrm{p}<0.001) \\
0.95(\mathrm{p}<0.001) \\
1.00(\mathrm{p}<0.001) \\
0.98(\mathrm{p}<0.001) \\
0.99(\mathrm{p}<0.001) \\
0.97(\mathrm{p}<0.001) \\
0.67(\mathrm{p}=0.20) \\
0.99(\mathrm{p}<0.001) \\
0.89(\mathrm{p}=0.003)\end{array}$ \\
\hline
\end{tabular}


Table 2. Cont.

\begin{tabular}{|c|c|c|c|c|c|c|c|}
\hline Reference & $\begin{array}{c}\text { Specimen } \\
\text { Type }\end{array}$ & Biomarker & Clinical Setting & Subjects & Sensitivity (\%) & Specificity (\%) & AUC \\
\hline $\begin{array}{l}\text { Park et al., } \\
2017 \text { [68] }\end{array}$ & Serum & $\begin{array}{c}\text { LRG1 + TTR + CA19-9 } \\
\text { CA19-9 } \\
\text { LRG1 + TTR + CA19-9 } \\
\text { CA19-9 } \\
\text { LRG1 + TTR + CA19-9 } \\
\text { CA19-9 } \\
\text { LRG1 + TTR + CA19-9 } \\
\text { CA19-9 } \\
\text { LRG1 + TTR + CA19-9 } \\
\text { CA19-9 } \\
\end{array}$ & $\begin{array}{c}80 \text { PDAC (50 stage I-II) (29 CA19-9 } \\
\text { negative PDAC), } 68 \text { HC, } 21 \text { BPD, } 52 \\
\text { Thyroid Ca, } 52 \text { Breast Ca, } 45 \\
\text { Colorectal Ca) }\end{array}$ & $\begin{array}{c}\text { PDAC vs. HC }+ \text { BPC }(n=89) \\
\text { PDAC stage I-II vs. HC }+ \text { BPC } \\
(n=89) \\
\text { PDAC vs. Other Cancers } \\
(n=149) \\
\text { PDAC vs. BPD } \\
\text { CA19-9 negative PDAC }(n=29) \\
\text { vs. HC + BPC }\end{array}$ & $\begin{array}{l}82.5 \\
72.5 \\
76.0 \\
64.0 \\
82.5 \\
72.5 \\
82.5 \\
72.5 \\
51.7 \\
24.1 \\
\end{array}$ & $\begin{array}{l}92.1 \\
88.8 \\
92.1 \\
88.8 \\
83.9 \\
87.9 \\
85.7 \\
81.0 \\
92.1 \\
88.8 \\
\end{array}$ & $\begin{array}{c}0.93(\mathrm{p}<0.01) \\
0.83 \\
0.91(\mathrm{p}<0.01) \\
0.79 \\
0.90(\mathrm{p}<0.001) \\
0.80 \\
0.90 \\
0.81 \\
0.83(\mathrm{p}<0.001) \\
0.52 \\
\end{array}$ \\
\hline $\begin{array}{l}\text { Arasaradnam } \\
\text { et al., } 2018 \text { [70] }\end{array}$ & Urine & Volatile organic compounds & $\begin{array}{c}4 \text { PDAC stage I, } 5 \text { stage IIA, } 35 \text { stage } \\
\text { IIB, } 24 \text { stage III, 12 stage IV, } \\
81 \mathrm{HC}\end{array}$ & $\begin{array}{c}\text { TS: PDAC vs. HC } \\
\text { VS: } \\
\text { PDAC vs. HC } \\
\text { PDAC stage I -II vs. HC } \\
\text { PDAC stage I-II vs. PDAC stage } \\
\text { III-IV }\end{array}$ & $\begin{array}{l}0.91 \\
0.90 \\
0.91 \\
0.82\end{array}$ & $\begin{array}{l}0.83 \\
0.81 \\
0.78 \\
0.89\end{array}$ & $\begin{array}{l}0.92(0.88-0.96) \\
0.92(0.85-0.98) \\
0.89(0.83-0.94) \\
0.92(0.86-0.97)\end{array}$ \\
\hline \multirow{4}{*}{$\begin{array}{l}\text { Dong et al., } \\
2018 \text { [71] }\end{array}$} & \multirow{4}{*}{ Serum } & $\begin{array}{c}\text { CA19-9 } \\
\text { POSTN } \\
\text { CA242 } \\
\text { CA19-9 + POSTN } \\
\text { CA19-9+ CA242 } \\
\text { POSTN+ CA242 } \\
\text { CA19-9 + POSTN+ CA242 }\end{array}$ & $\begin{array}{c}30 \text { PDAC (early stage), } \\
68 \text { PDAC (late stage), } \\
32 \text { BPC, } \\
37 \text { HC, } \\
27 \text { PDAC (CA19-9 negative) }\end{array}$ & $\begin{array}{c}\text { TS: } \\
\text { HC vs. early PDAC }\end{array}$ & $\begin{array}{l}96.7 \\
70.0 \\
81.1 \\
93.3 \\
90.0 \\
83.3 \\
96.7\end{array}$ & $\begin{array}{l}83.8 \\
75.7 \\
81.1 \\
94.6 \\
94.6 \\
86.5 \\
94.6\end{array}$ & $\begin{array}{l}0.94(0.86-0.99) \\
0.78(0.66-0.87) \\
0.89(0.79-0.95) \\
0.97(0.90-1.00) \\
0.96(0.88-0.99) \\
0.90(0.80-0.96) \\
0.98(0.90-1.00)\end{array}$ \\
\hline & & $\begin{array}{c}\text { CA19-9 } \\
\text { POSTN } \\
\text { CA242 } \\
\text { CA19-9 + POSTN } \\
\text { CA19-9+ CA242 } \\
\text { POSTN+ CA242 } \\
\text { CA19-9 + POSTN+ CA242 }\end{array}$ & & BPC vs. all PDAC & $\begin{array}{l}85.7 \\
64.3 \\
58.1 \\
84.7 \\
75.5 \\
67.4 \\
84.7\end{array}$ & $\begin{array}{l}81.3 \\
87.5 \\
87.5 \\
90.6 \\
90.6 \\
96.9 \\
90.6\end{array}$ & $\begin{array}{l}0.88(0.82-0.93) \\
0.81(0.74-0.88) \\
0.78(0.70-0.85) \\
0.93(0.88-0.97) \\
0.89(0.83-0.94) \\
0.87(0.80-0.92) \\
0.94(0.88-0.97)\end{array}$ \\
\hline & & $\begin{array}{c}\text { CA19-9 } \\
\text { POSTN } \\
\text { CA242 } \\
\text { CA19-9 + POSTN } \\
\text { CA19-9+ CA242 } \\
\text { POSTN+ CA242 } \\
\text { CA19-9 + POSTN+ CA242 }\end{array}$ & $\begin{array}{c}38 \text { PDAC (early stage), } \\
77 \text { PDAC (late stage), } \\
43 \text { BPC; } \\
37 \text { HC, } \\
29 \text { PDAC (CA19-9 negative) }\end{array}$ & BPC vs. early PDAC & $\begin{array}{l}86.7 \\
53.3 \\
83.3 \\
96.7 \\
90.0 \\
56.7 \\
96.7\end{array}$ & $\begin{array}{l}81.3 \\
87.5 \\
62.5 \\
75.0 \\
78.1 \\
96.9 \\
75.0\end{array}$ & $\begin{array}{l}0.88(0.77-0.95) \\
0.74(0.61-0.84) \\
0.78(0.66-0.88) \\
0.90(0.80-0.96) \\
0.90(0.79-0.96) \\
0.80(0.68-0.89) \\
0.92(0.82-0.97)\end{array}$ \\
\hline & & $\begin{array}{c}\text { CA19-9 } \\
\text { POSTN } \\
\text { CA242 } \\
\text { CA19-9 + POSTN } \\
\text { CA19-9+ CA242 } \\
\text { POSTN+ CA242 } \\
\text { CA19-9 + POSTN+ CA242 }\end{array}$ & & $\begin{array}{c}\text { VS: } \\
\text { HC vs. early PDAC }\end{array}$ & $\begin{array}{l}86.8 \\
63.2 \\
57.9 \\
86.8 \\
86.8 \\
79.0 \\
92.1\end{array}$ & $\begin{array}{l}94.6 \\
78.4 \\
100 \\
97.3 \\
97.3 \\
94.6 \\
97.3\end{array}$ & $\begin{array}{l}0.94(0.86-0.98) \\
0.78(0.66-0.86) \\
0.83(0.73-0.91) \\
0.95(0.88-0.99) \\
0.97(0.90-1.00) \\
0.92(0.84-0.97) \\
0.98(0.92-1.00)\end{array}$ \\
\hline
\end{tabular}


Table 2. Cont.

\begin{tabular}{|c|c|c|c|c|c|c|c|}
\hline Reference & $\begin{array}{l}\text { Specimen } \\
\text { Type }\end{array}$ & Biomarker & Clinical Setting & Subjects & Sensitivity (\%) & Specificity (\%) & AUC \\
\hline \multirow[t]{2}{*}{ Dong et al., 2018 [71] } & \multirow[t]{2}{*}{ Serum } & $\begin{array}{c}\text { CA19-9 } \\
\text { POSTN } \\
\text { CA242 } \\
\text { CA19-9+ POSTN } \\
\text { CA19-9 + CA242 } \\
\text { POSTN+ CA242 } \\
\text { CA19-9 + POSTN+ CA242 }\end{array}$ & & $\mathrm{BPC}$ vs. all PDAC & $\begin{array}{l}84.4 \\
77.4 \\
60.0 \\
83.5 \\
77.4 \\
84.4 \\
80.0\end{array}$ & $\begin{array}{l}81.4 \\
79.1 \\
93.0 \\
93.0 \\
88.4 \\
83.7 \\
97.7\end{array}$ & $\begin{array}{l}0.88(0.82-0.93) \\
0.82(0.76-0.88) \\
0.79(0.72-0.85) \\
0.92(0.87-0.96) \\
0.90(0.84-0.94) \\
0.89(0.83-0.93) \\
0.93(0.88-0.96)\end{array}$ \\
\hline & & $\begin{array}{c}\text { CA19-9 } \\
\text { POSTN } \\
\text { CA242 } \\
\text { CA19-9+ POSTN } \\
\text { CA19-9+ CA242 } \\
\text { POSTN+ CA242 } \\
\text { CA19-9 + POSTN+ CA242 }\end{array}$ & & $\begin{array}{c}\text { BPC vs. early } \\
\text { PDAC }\end{array}$ & $\begin{array}{l}86.8 \\
65.8 \\
57.9 \\
65.8 \\
76.3 \\
76.3 \\
83.6\end{array}$ & $\begin{array}{l}79.1 \\
79.1 \\
93.0 \\
93.0 \\
93.0 \\
86.1 \\
88.4\end{array}$ & $\begin{array}{l}0.87(0.78-0.93) \\
0.72(0.61-0.82) \\
0.77(0.66-0.85) \\
0.84(0.75-0.92) \\
0.92(0.83-0.97) \\
0.84(0.74-0.91) \\
0.90(0.81-0.96)\end{array}$ \\
\hline Guo et al., 2018 [72] & Serum & SNHG15 & $171 \mathrm{PDAC}, 59 \mathrm{HC}$ & PDAC vs. HC & 68.3 & 89.6 & $0.73(\mathrm{p}<0.01)$ \\
\hline Lewis et al., 2018 [73] & $\begin{array}{l}\text { Whole blood, } \\
\text { plasma, serum }\end{array}$ & GPC1 + CD63 & $\begin{array}{c}20 \text { PDAC } \\
6 \mathrm{BPD} \\
\end{array}$ & PDAC vs. BPD & 81 & 70 & $0.79(0.99-1.00)$ \\
\hline \multirow[t]{2}{*}{ Mellby et al., 2018 [74] } & \multirow[t]{2}{*}{ Plasma } & \multirow[t]{2}{*}{ Panel of 29 biomarkers } & \multirow{2}{*}{$\begin{array}{c}15 \text { PDAC stage I, } \\
75 \text { stage II, } 15 \text { stage III, } 38 \text { stage IV } \\
57 \text { CP, } \\
20 \text { IPMN, } \\
219 \mathrm{HC}\end{array}$} & $\begin{array}{c}\text { TS 2: } \\
\text { PDAC stage I-II } \\
\text { vs. HC }\end{array}$ & 95 & 94 & $0.96(0.94-0.98)$ \\
\hline & & & & $\begin{array}{l}\text { VS (USA cohort): } \\
\text { PDAC stage I-II } \\
\text { vs. HC }\end{array}$ & 93 & 95 & $0.96(0.94-0.98)$ \\
\hline Traeger et al., 2018 [75] & Serum & $\begin{array}{c}\text { miRNA-205 } \\
\text { CA19-9 } \\
\text { miRNA-205 +CA19-9 }\end{array}$ & $\begin{array}{l}47 \text { PDAC, } \\
16 \text { CP, } \\
5 \text { IPMN, } \\
17 \text { BPC, } \\
17 \text { HC }\end{array}$ & $\begin{array}{l}\text { PDAC vs. } \\
\text { non-PDAC }\end{array}$ & $\begin{array}{l}0.643 \\
0.810 \\
0.867\end{array}$ & $\begin{array}{l}0.684 \\
0.768 \\
0.933\end{array}$ & $\begin{array}{l}0.70(0.548-0.789 \\
0.79(0.698-0.887 \\
0.89(0.782-0.995)\end{array}$ \\
\hline \multirow{4}{*}{ Zhou et al., 2018 [76] } & \multirow{4}{*}{ Serum } & $\begin{array}{c}\text { GPC1 } \\
\text { CA19-9 }\end{array}$ & $\begin{array}{l}156 \mathrm{PDAC}, \\
20 \mathrm{BPT}, \\
16 \mathrm{CP}, \\
163 \mathrm{HC}\end{array}$ & $\begin{array}{c}\text { PDAC vs. HC + } \\
\text { BPT + CP }\end{array}$ & $\begin{array}{l}76.92 \\
82.69\end{array}$ & $\begin{array}{l}70.85 \\
93.97\end{array}$ & $\begin{array}{l}0.80(0.749-0.841 \\
0.91(0.868-0.947)\end{array}$ \\
\hline & & $\begin{array}{c}\text { GPC1 } \\
\text { CA19-9 }\end{array}$ & & PDAC vs. HC & $\begin{array}{l}76.92 \\
82.69\end{array}$ & $\begin{array}{l}70.55 \\
97.55\end{array}$ & $\begin{array}{l}0.81(0.763-0.856 \\
0.91(0.875-0.953)\end{array}$ \\
\hline & & $\begin{array}{c}\text { GPC1 } \\
\text { CA19-9 }\end{array}$ & & $\begin{array}{l}\text { Early PDAC vs. } \\
\text { HC + BPT + CP }\end{array}$ & $\begin{array}{l}68.06 \\
79.17\end{array}$ & $\begin{array}{l}70.85 \\
93.97\end{array}$ & $\begin{array}{l}0.76(0.695-0.816 \\
0.88(0.816-0.946)\end{array}$ \\
\hline & & $\begin{array}{c}\text { GPC1 } \\
\text { CA19-9 }\end{array}$ & & $\begin{array}{c}\text { Early PDAC vs. } \\
\text { HC }\end{array}$ & $\begin{array}{l}68.06 \\
79.17\end{array}$ & $\begin{array}{l}70.55 \\
97.55\end{array}$ & $\begin{array}{l}0.77(0.705-0.830 \\
0.89(0.824-0.953)\end{array}$ \\
\hline
\end{tabular}


Table 2. Cont

\begin{tabular}{|c|c|c|c|c|c|c|c|}
\hline Reference & $\begin{array}{c}\text { Specimen } \\
\text { Type }\end{array}$ & Biomarker & Clinical Setting & Subjects & Sensitivity (\%) & Specificity (\%) & AUC \\
\hline \multirow{2}{*}{ Berger et al., 2019 [77] } & \multirow[b]{2}{*}{ Plasma } & $\begin{array}{c}\text { CA19-9 } \\
\text { THBS2 } \\
\text { cfDNA } \\
\text { CA19-9 + THBS2 } \\
\text { THBS2 + CA19-9 + cfDNA }\end{array}$ & $\begin{array}{l}52 \text { PDAC, } \\
15 \text { IPMN, } \\
32 \mathrm{CP}\end{array}$ & $\begin{array}{c}\text { TS: } \\
\text { PDAC vs. IPMN vs. CP }\end{array}$ & $\begin{array}{c}55 \\
41 \\
32-86^{1} \\
73 \\
41-86^{1}\end{array}$ & $\begin{array}{c}91 \\
96 \\
70-100^{1} \\
91 \\
78-96^{1}\end{array}$ & $\begin{array}{l}0.80 \\
0.73 \\
0.90 \\
0.87 \\
0.94\end{array}$ \\
\hline & & $\begin{array}{c}\text { CA19-9 } \\
\text { THBS2 } \\
\text { cfDNA } \\
\text { CA19-9 + THBS2 } \\
\text { THBS2 + CA19-9 + cfDNA }\end{array}$ & & $\begin{array}{c}\text { VS: } \\
\text { PDAC vs. IPMN vs. CP }\end{array}$ & $\begin{array}{c}63 \\
50 \\
43-80^{1} \\
80 \\
50-93^{1} \\
\end{array}$ & $\begin{array}{c}96 \\
96 \\
79-96^{1} \\
96 \\
92-96^{1}\end{array}$ & $\begin{array}{l}0.70 \\
0.63 \\
0.81 \\
0.78 \\
0.88\end{array}$ \\
\hline \multirow{3}{*}{ Eissa et al., 2019 [78] } & \multirow{3}{*}{ Plasma } & ADAMTS1 & $\begin{array}{l}39 \text { PDAC, } \\
95 \mathrm{HC}\end{array}$ & PDAC vs. HC & 87.2 & 95.8 & $0.91(0.77-0.90)$ \\
\hline & & $\mathrm{BNC1}$ & & & 64.1 & 93.7 & $0.79(0.63-0.78)$ \\
\hline & & ADAMTS1 and/or BNC1 & & & 97.4 & 91.6 & $0.95(0.71-0.86)$ \\
\hline \multirow{3}{*}{$\begin{array}{l}\text { Fahrmann et al., } \\
\quad 2019[79]\end{array}$} & \multirow{3}{*}{ Plasma } & $\begin{array}{l}\text { AcSperm+ DAS + indole-derivative+ } \\
\text { LysoPC(18:0) + LysoPC(20:3) }\end{array}$ & $\begin{array}{l}29 \text { PDAC, } \\
10 \mathrm{HC}\end{array}$ & $\begin{array}{c}\text { TS: } \\
\text { PDAC vs. HC }\end{array}$ & 69.0 & 99.0 & $0.90(0.818-0.989)$ \\
\hline & & $\begin{array}{l}\text { AcSperm+ DAS + indole-derivative+ } \\
\text { LysoPC(18:0) + LysoPC(20:3) }\end{array}$ & $\begin{array}{l}39 \text { Resectable PDAC, } \\
82 \mathrm{HC}\end{array}$ & $\begin{array}{c}\text { VS: } \\
\text { PDAC vs. HC }\end{array}$ & 66.7 & 43.3 & $0.89(0.828-0.996)$ \\
\hline & & $\begin{array}{c}\text { Indole-derivative } \\
\text { LysoPC }(18: 0) \\
\text { LysoPC }(20: 3) \\
\text { AcSperm } \\
\text { DAS }\end{array}$ & & & $\begin{array}{l}23.1 \\
51.3 \\
48.7 \\
33.3 \\
51.3\end{array}$ & $\begin{array}{l}11.3 \\
26.3 \\
11.3 \\
27.5 \\
27.5\end{array}$ & $\begin{array}{l}0.73(0.631-0.822) \\
0.84(0.764-0.920) \\
0.840 .757-0.925) \\
0.76(0.659-0.852) \\
0.80(0.712-0.890)\end{array}$ \\
\hline Yu et al., 2019 [80] & Plasma & $\begin{array}{l}\text { d-signature: EV long RNA (FGA, } \\
\text { KRT19, HIST1H2BK, ITIH2, } \\
\text { MARCH2, CLDN1, MAL2 and } \\
\text { TIMP1) }\end{array}$ & 284 PDAC, 100 CP, 117 HC & $\begin{array}{l}\text { PDAC vs. CP vs. HC } \\
\text { d-signature with } \\
\text { CA19-9 }\end{array}$ & 93.68 & 91.57 & $0.936(0.889-0.983)$ \\
\hline $\begin{array}{l}\text { Takahashi et al., } \\
2019[81]\end{array}$ & Serum & Circulating EV-encapsulated HULC & 20 PDAC, 22 IPMN, 21 HC & PDAC vs. IPMN vs. HC & 80 & 92.1 & 0.92 \\
\hline Wei et al., 2019 [82] & Plasma & Vimetin-positive CTCs & 100 PDAC, 16 IPMN, 30 HC & PDAC vs. IPMN vs. HC & 65 & 100 & 0.968 \\
\hline Yang et al., 2020 [83] & Plasma & $\begin{array}{c}\text { EV miRNAs and mRNAs, cfDNA, } \\
\text { ccfDNA KRAS G12D/V/R } \\
\text { mutations and CA19-9 }\end{array}$ & $\begin{array}{c}30 \mathrm{CP}+\mathrm{BPC}, 49 \text { PDAC, } 57 \\
\text { HC }\end{array}$ & $\begin{array}{l}\text { PDAC vs. non-PDAC } \\
\text { vs. HC }\end{array}$ & 88 & 95 & $0.95(\mathrm{p}=0.103)$ \\
\hline
\end{tabular}

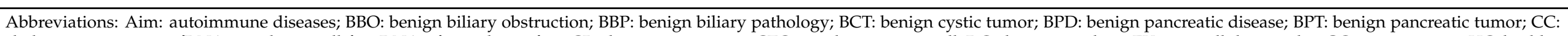

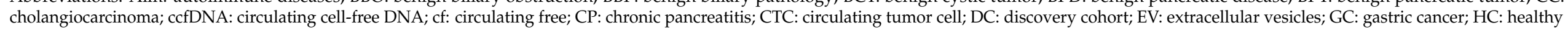

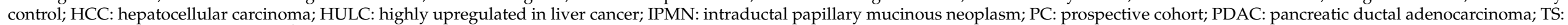
training set; VS: validation set. Note: ${ }^{1}$ Depending on the concentration of cfDNA. 
The studies summarized in Table 2 were all aimed at earlier PDAC detection; however, around $20 \%$ of them did not analyze their biomarkers in resectable stage I-II samples. While the paucity of earliest stage I samples is a recognized problem in the PDAC field, this was surprising, considering the critical importance of diagnostic stage shift. Selection of the relevant control population is also of the essence, as PDAC is an uncommon cancer, which dictates the need to assess the putative biomarkers in high-risk groups $[1,84,85]$, a criterion which was not satisfied in $20 \%$ of the studies. It also requires the high accuracy of any potential future biomarker assay with at least $88 \% \mathrm{SN}$ and $85 \%$ SP if the test is to be cost-effective [86].

A third of the reviewed studies did not include a comparison of their biomarkers to CA19-9, which was unexpected considering that CA19-9 is the most commonly measured biomarker in PDAC patients. Very few biomarkers were conclusively superior to CA19-9 and in the majority of studies where their combination was tested, the accuracy of PDAC detection was superior to either biomarker alone. For example, Dong et al. [71] described the panel of serum biomarkers comprising CA19-9, POSTN and CA242, which was superior to CA19-9 alone in differentiating between PDAC and both healthy controls (AUC of 0.98, $92.1 \% \mathrm{SN}$ and $97.3 \% \mathrm{SP}$ ) and benign pancreatic conditions (AUC of $0.93,80 \% \mathrm{SN}$ and $97.7 \%$ SP), albeit with somewhat lower AUC of 0.90 and a decrease in SP to $88 \%$ in resectable PDAC. In the study of Berger et al. [77], CA19-9 combined with THBS2 and cfDNA in PDAC vs. non-PDAC patients showed an increase in AUC from 0.70 to 0.78, with an increased SN and SP of $80 \%$ and $96 \%$, respectively. A study by Fahrmann et al. [79] combined a five-metabolite panel (acetylspermidine, diacetylspermine, an indole-derivative and two lysophosphatidylcholines) with a previously validated protein panel comprising CA19-9, TIMP1 and LRG1, achieving AUC of 0.924 (95\% CI 0.864-0.983). Recently, another panel (FGA, KRT19, HIST1H2BK, ITIH2, MARCH2, CLDN1, MAL2 and TIMP1) obtained by plasma extracellular vesicle long RNA profiling (d-signature), successfully differentiated PDAC from CP and healthy controls with AUC of 0.936, and both SN and SP > 90\%; furthermore, when combined with CA19-9, the signature showed improved AUC of 0.964 (95\% CI: 0.943-0.984) [80]. Traeger et al., reported that miRNA-205 combined with CA19-9 resulted in higher SN and SP in comparison of PDAC vs. benign specimens $(86.7 \% \mathrm{SN}$ and 93.3\% SP) than either of the biomarkers alone [75].

The absolute SN and SP values for PDAC detection using glypican-1 (GPC1) in circulating exosomes when PDAC samples were compared to healthy and benign samples was reported by Melo et al. [54]. However, several subsequent studies failed to support these findings $[62,73,87,88]$, while Zhou et al. [76] demonstrated that combining GPC1 and CA19-9 showed $92.31 \%$ SN at $65.83 \%$ SP for distinguishing healthy, chronic pancreatitis and benign pancreatic tumors from PDAC. Regardless of the underlying reasons, these conflicting results highlight the critical importance of independent validation of any proposed biomarkers.

The above study [54] and the more recent studies indicate the "expansion" of the biomarker field, moving from the "traditional" DNA, RNA and protein biomarkers to novel, versatile analytes and their combinations, such as non-coding RNAs, epigenetic markers, lipids and metabolites, as well as circulating tumor cells and extracellular vesicles (EV) [88]. For example, Yang et al. [83] performed a multi-parametric analysis of blood tumor-associated EV miRNA and mRNA, circulating cell-free DNA concentration and KRAS G12D/V/R mutations with CA19-9, and demonstrated their higher accuracy for PDAC staging (84\%) than imaging alone (accuracy $=64 \% ; \mathrm{P}<0.05$ ). Furthermore, recent advances in computer science, machine learning and artificial intelligence (AI) have given rise to the emerging field of bioinformatics and computational biology which allow analyses of large collections of biological data [89]. While AI was mostly utilized for the prediction of risk/diagnosis based on health records or abdominal imaging [1,90,91], it also shows a great potential for in silico discovery of putative novel biomarkers. For example, Khatri and Bhasin [92] used transcriptomics-based meta-analysis of tissue and blood samples combined with machine learning to identify a nine-gene panel (IFI27, ITGB5, CTSD, EFNA4, 
GGH, PLBD1, HTATIP2, IL1R2 and CTSA) that differentiated PDAC and healthy controls with $92 \%$ SN and 90\% SP. Moreover, this panel also discriminated PDAC from chronic pancreatitis and early precursor lesions in non-malignant tissue and peripheral blood. Chung et al. [93] used probe electrospray ionization mass spectrometry (PESI-MS) and a machine learning approach to analyze peripheral blood samples and achieved an accuracy of $92.1 \%$ in healthy vs. PDAC $(90.8 \%$ SN, $91.7 \%$ SP). The combination of PESI-MS profiles with age and CA19-9 increased the accuracy for detection of stage I and II PDAC to $92.9 \%$ $(81.2 \% \mathrm{SN}, 96.8 \% \mathrm{SP})$ [40]. This demonstrates that the access to richly annotated patients health records will enable the construction of novel PDAC prediction tools such as the one developed by Muhammad et al. [90]. Combining such prediction tools with biofluid biomarkers now opens up promising new avenues and likely a fruitful approach to identify asymptomatic patients at increased risk of developing PDAC.

Very few studies explored the effectiveness of their proposed biomarkers in prediagnostic samples from asymptomatic patients. Although such samples are not readily available, these studies are essential for determining how early in the latency period the biomarkers can be detected [40,47,94-101]. Nolen et al. [47] interrogated the large prospective Prostate, Lung, Colorectal, and Ovarian (PLCO) Cancer Screening Trial serum samples to detect eight biomarkers: CEA-related cell adhesion molecule 1 (CEACAM1) and prolactin (PRL) were detectable up to 35 months before PDAC diagnosis; CA19-9, carcinoembryonic antigen (CEA), neuron-specific enolase (NSE) and beta-human chorionic gonadotropin (b-HCG) up to 24 months; and CA125 and interleukin 8 (IL-8) up to 12 months prior PDAC diagnosis. In the UK Collaborative Trial of Ovarian Cancer Screening (UKCTOCS) serum samples, a decrease in circulating TSP-1 levels up to 24 months prior to PDAC diagnosis was described by Jenkinson et al. [97], and O'Brien et al. demonstrated that CA19-9 could be detected in such pre-diagnostic PDAC samples with $95 \% \mathrm{SP}$ and $68 \%$ $\mathrm{SN}$ up to 1 year and $53 \% \mathrm{SN}$ up to 2 years prior to PDAC diagnosis [98]. A three-biomarker panel comprising Erb-B2 receptor tyrosine kinase 2 (ERBB2), estrogen receptor 1 (ESR1) and tenascin C (TNC) was analyzed in 87 pre-diagnostic plasma samples from Women's Health Initiative (WHI) collected up to 4 years before diagnosis and achieved AUC of 0.71 when combined with CA19-9 [100]. Recently, the analysis of 1196 proteins in longitudinal plasma samples from the Institute for Systems Biology's 100K Wellness project identified several markers, including three that were observed in PDAC: CEA cell adhesion molecule 5 (CEACAM5) was expressed at high levels 26.5 months pre-diagnosis, while calcitonin related polypeptide alpha (CALCA) and delta1 like homolog (DLK1) were predating PDAC diagnosis for around 17 months [101]. More exciting projects such as this are needed and should be actively encouraged.

While performing this review, we encountered a number of hurdles, particularly noticeable in the way the studies were reported. Easier reviewing of published resources would be facilitated, and the extraction of the relevant information simplified, if a consensus set of standards for the reporting of biomarker studies were utilized. In addition to QUADAS-2, several of such recommendations and guidelines exist: BRISQ (Biospecimen Reporting for Improved Study Quality) [102] provides recommendations for reporting details about the interrogated biospecimens; the Standards for Reporting of Diagnostic Accuracy Studies (STARD) [103] was developed to improve the completeness and transparency of the reports; and Reporting Recommendations for Tumour Marker Prognostic Studies (REMARK) [104] and Consolidated Standards of Reporting Trials (CONSORT Statement) [105] are used to improve the overall quality of tumor markers in the prognostic studies and randomized trials $[104,105]$. The latter is the final step in establishing the clinical utility of the biomarker, i.e., increased survival due to earlier diagnosis achieved by biomarker testing. Interestingly, only studies published in high-impact factors journals followed any of these recommendations $[49,54,66,74,80]$.

In addition to the standardized way of reporting, a standardized pathway for early detection and diagnosis is also needed. Such pathway has recently been summarized in the updated Cancer Research UK (CRUK) roadmap, which aims to consolidate the 
current diverse and fragmented research activities, in line with the future vision to detect $75 \%$ of cancers at stage I-II by 2028 [106]. A roadmap also illustrates a long way from biomarker discovery to its implementation, with a number of hurdles encountered at every step in biomarker pipeline, from initial biomarker discovery, its quantification, verification and assay optimization to finally biomarker validation and commercialization, as comprehensively discussed previously [107-109]. While biomarker discovery is usually well funded and results straightforward to report, validation, which is a necessary step prior to commercialization, is time-consuming and expensive process, with scarce funding available. The transition from discovery to the clinical utility of any biomarker is therefore a cumbersome process, akin to the implementation of novel therapeutic [110].

It is not surprising thus that, despite a large number of candidate biomarkers, very few are close to clinical implementation. Based on the accuracy of $95 \%$ of serum biomarker signature published by Mellby et al. [74] and using their proprietary antibody platform, Immunovia is now developing the commercial assay IMMRay ${ }^{\mathrm{TM}}$ PanCan- $\mathrm{d}$, which is currently being further validated in the prospective studies [111]. Our urinary biomarker panel comprising REG1B, LYVE1, TFF1 and the affiliated PancRISK score [112,113] are now being validated in a prospective study UroPanc [114] . A multi-analyte blood test that simultaneously evaluates the levels of eight cancer proteins and the presence of cancer gene mutations from circulating DNA, CancerSEEK ${ }^{\circledR}$, screens for eight common cancers, including PDAC [115], and has already been commercialized. GRAIL is advocating its own Galleri test, which is based on cfDNA methylation signal and can detect over 50 different cancers [116]. Both platforms, however, suffer from lower SN in detecting stage I cancers, and there is a legitimate concern that the tests might be less specific in real-world conditions, where comorbidities may increase the risk of a false-positive result [117].

\section{Conclusions}

In summary, our systematic review demonstrates the current progress in the biomarker field and highlights several promising non-invasive PDAC biomarkers. Most of these, however, still require further validation prior to being deemed appropriate for translation into the clinical setting. All future studies should aim to improve methodological quality and the way of reporting. Novel biomarkers that have shown promising results should be further investigated on a larger number of specimens, as well as in a pre-diagnostic and prospective setting, to further demonstrate their real clinical utility in earlier detection of pancreatic adenocarcinoma.

Author Contributions: Conceptualization, V.S., G.B. and T.C.-J.; methodology, G.B., V.S. and T.C.-J.; writing and editing, G.B., V.S., D.J. and T.C.-J.; and supervision, T.C.-J. All authors have read and agreed to the published version of the manuscript.

Funding: This research received no external funding.

Conflicts of Interest: The authors declare no conflict of interest.

\section{Appendix A}

Table A1. Graphical summary of the QUADAS-2 scores for each of the 49 studies.

\begin{tabular}{|c|c|c|c|c|c|c|c|c|}
\hline \multirow[b]{2}{*}{ Nr. } & \multirow[b]{2}{*}{ Study } & \multicolumn{3}{|c|}{ Risk of Bias } & \multicolumn{3}{|c|}{ Applicability Concerns } & \multirow[b]{2}{*}{ Score } \\
\hline & & $\begin{array}{l}\text { Patient } \\
\text { Selection }\end{array}$ & $\begin{array}{c}\text { Index } \\
\text { Test }\end{array}$ & $\begin{array}{l}\text { Reference } \\
\text { Standard }\end{array}$ & $\begin{array}{l}\text { Patient } \\
\text { Selection }\end{array}$ & $\begin{array}{c}\text { Index } \\
\text { Test }\end{array}$ & $\begin{array}{l}\text { Reference } \\
\text { Standard }\end{array}$ & \\
\hline 1 & Gold et al., 2010 [37] & (:) & $?$ & $?$ & (:) & (:) & (:) & Low \\
\hline 2 & Joergensen et al., 2010 [38] & (:) & $?$ & (:) & (:) & (:) & (:) & Low \\
\hline 3 & Marten et al., 2010 [39] & (:) & $?$ & (:) & (:) & $?$ & (:) & High \\
\hline 4 & Brand et al., 2011 [17] & (-) & (); & $?$ & (:) & () & (:) & Low \\
\hline
\end{tabular}


Table A1. Cont.

\begin{tabular}{|c|c|c|c|c|c|c|c|c|}
\hline \multirow[b]{2}{*}{ Nr. } & \multirow[b]{2}{*}{ Study } & \multicolumn{3}{|c|}{ Risk of Bias } & \multicolumn{3}{|c|}{ Applicability Concerns } & \multirow[b]{2}{*}{ Score } \\
\hline & & $\begin{array}{c}\text { Patient } \\
\text { Selection }\end{array}$ & $\begin{array}{c}\text { Index } \\
\text { Test }\end{array}$ & $\begin{array}{l}\text { Reference } \\
\text { Standard }\end{array}$ & $\begin{array}{c}\text { Patient } \\
\text { Selection }\end{array}$ & $\begin{array}{c}\text { Index } \\
\text { Test }\end{array}$ & $\begin{array}{l}\text { Reference } \\
\text { Standard }\end{array}$ & \\
\hline 5 & Park et al., 2012 [21] & $\odot$ & $?$ & () & (-) & (-) & (:) & Low \\
\hline 6 & Capello et al., 2013 [40] & $\odot$ & () & $\odot$ & () & $\odot$ & (-) & Low \\
\hline 7 & Gold et al., 2013 [41] & $\odot$ & $?$ & () & ()$^{\circ}$ & () & $\odot$ & Low \\
\hline 8 & Kobayashi et al., 2013 [42] & () & () & $\odot$ & () & () & () & Low \\
\hline 9 & Li et al., 2013 [43] & (-) & $\odot$ & () & $\odot$ & () & $\odot$ & Low \\
\hline 10 & Zhao et al., 2013 [44] & () & $(\odot$ & $?$ & $\odot$ & $\odot$ & $\odot$ & High \\
\hline 11 & Chung et al., 2014 [45] & $\odot$ & $\odot$ & () & $\odot$ & $\odot$ & $\odot$ & Low \\
\hline 12 & Lee et al., 2014 [46] & (-) & $\odot$ & (:) & () & () & $\odot$ & Low \\
\hline 13 & Nolen et al., 2014 [47] & $\odot$ & $\odot$ & () & (:) & () & (-) & Low \\
\hline 14 & Ren et al., 2014 [48] & () & $\odot$ & () & ()$^{\circ}$ & () & $\odot$ & Low \\
\hline 15 & Schultz et al., 2014 [49] & (-) & $?$ & () & $\odot$ & (-) & $\odot$ & Low \\
\hline 16 & Yang et al., 2014 [50] & (-) & (-) & $?$ & () & $?$ & $\odot$ & High \\
\hline 17 & Zhang et al., 2014 [51] & $\odot$ & $?$ & $\odot$ & $\odot$ & () & $\odot$ & Low \\
\hline 18 & Debernardi et al., 2015 [52] & (:) & $?$ & $?$ & $\odot$ & () & $\odot$ & High \\
\hline 19 & Han et al., 2015 [53] & $\odot$ & (-) & $\odot$ & (-) & () & (:) & Low \\
\hline 20 & Melo et al., 2015 [54] & $\odot$ & $?$ & $\odot$ & $\odot$ & $\odot$ & (-) & Low \\
\hline 21 & Radon et al., 2015 [55] & () & $?$ & (;) & () & (;) & (-) & Low \\
\hline 22 & Ankeny et al., 2016 [56] & $\odot$ & () & $?$ & $\odot$ & $?$ & () & High \\
\hline 23 & Guo et al., 2016 [57] & $\odot$ & () & $\odot$ & (:) & () & (-) & Low \\
\hline 24 & Henriksen et al., 2016 [58] & () & () & $?$ & $\odot$ & () & (-) & Low \\
\hline 25 & Sogawa et al., 2016 [59] & $\odot$ & (-) & () & () & () & $\odot$ & Low \\
\hline 26 & Yoneyama et al., 2016 [60] & (-) & $?$ & () & () & () & (-) & Low \\
\hline 27 & Balasenthil et al., 2017 [61] & $\odot$ & (-) & () & $\odot$ & () & () & Low \\
\hline 28 & Yang et al., 2017 [62] & (-) & $?$ & () & $\odot$ & $?$ & () & Low \\
\hline 29 & Capello et al., 2017 [63] & $\odot$ & $?$ & $\odot$ & $\odot$ & $\odot$ & $\odot$ & Low \\
\hline 30 & Hussein et al., 2017 [64] & (-) & $\odot$ & () & () & () & $\odot$ & High \\
\hline 31 & Kaur et al., 2017 [65] & $\odot$ & $\odot$ & () & $\odot$ & 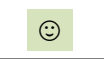 & $\odot$ & Low \\
\hline 32 & Kim et al., 2017 [66] & (-) & $\odot$ & () & $\odot$ & () & () & Low \\
\hline 33 & Lai et al., 2017 [67] & (-) & () & () & () & () & $\odot$ & High \\
\hline 34 & Park et al., 2017 [68] & $\odot$ & (-) & $\odot$ & $\odot$ & $\odot$ & $\odot$ & Low \\
\hline 35 & Schott et al., 2017 [69] & () & () & $?$ & $\odot$ & $\odot$ & $\odot$ & High \\
\hline 36 & Arasaradnam et al., 2018 [70] & (-) & $?$ & $?$ & $\odot$ & () & $\odot$ & High \\
\hline 37 & Dong et al., 2018 [71] & (-) & $?$ & $\odot$ & $\odot$ & () & $\odot$ & Low \\
\hline 38 & Guo et al., 2018 [72] & (:) & () & $?$ & (-) & () & $\odot$ & High \\
\hline
\end{tabular}


Table A1. Cont.

\begin{tabular}{|c|c|c|c|c|c|c|c|c|}
\hline \multirow[b]{2}{*}{ Nr. } & \multirow[b]{2}{*}{ Study } & \multicolumn{3}{|c|}{ Risk of Bias } & \multicolumn{3}{|c|}{ Applicability Concerns } & \multirow[b]{2}{*}{ Score } \\
\hline & & $\begin{array}{c}\text { Patient } \\
\text { Selection }\end{array}$ & $\begin{array}{c}\text { Index } \\
\text { Test }\end{array}$ & $\begin{array}{l}\text { Reference } \\
\text { Standard }\end{array}$ & $\begin{array}{c}\text { Patient } \\
\text { Selection }\end{array}$ & $\begin{array}{c}\text { Index } \\
\text { Test }\end{array}$ & $\begin{array}{l}\text { Reference } \\
\text { Standard }\end{array}$ & \\
\hline 39 & Mellby et al., 2018 [74] & (); & $?$ & (); & (:) & (); & () & Low \\
\hline 40 & Traeger et al., 2018 [75] & $\odot$ & (2) & (-) & ;) & (-) & () & Low \\
\hline 41 & Zhou et al., 2018 [76] & (:) & (:) & ;) & (2) & $?$ & () & High \\
\hline 42 & Berger et al., 2019 [77] & () & (;) & () & () & (:) & (;) & Low \\
\hline 43 & Eissa et al., 2019 [78] & (:) & (:) & $?$ & (:) & (:) & (:) & Low \\
\hline 44 & Fahrmann et al., 2019 [79] & (:) & (:) & (:) & (:) & (:) & (:) & Low \\
\hline 45 & Lewis et al., 2019 [73] & (-) & $?$ & $?$ & (:) & $?$ & ;) & High \\
\hline 46 & Yu et al., 2019 [80] & (:) & (:) & (:) & (:) & () & (:) & Low \\
\hline 47 & Takahashi et al., 2019 [81] & ;) & (:) & $?$ & (:) & $?$ & ;) & High \\
\hline 48 & Wei et al., 2019 [82] & (:) & (:) & (); & (:) & $?$ & (); & Low \\
\hline 49 & Yang et al., 2020 [83] & (:) & () & (:) & (:) & $?$ & (:) & Low \\
\hline
\end{tabular}

(-) Low Risk; $\odot$ High Risk; ? Unclear Risk.

\section{References}

1. Pereira, S.P.; Oldfield, L.; Ney, A.; Hart, P.A.; Keane, M.G.; Pandol, S.J.; Li, D.; Greenhalf, W.; Jeon, C.Y.; Koay, E.J.; et al. Early detection of pancreatic cancer. Lancet Gastroenterol. Hepatol. 2020, 5, 698-710. [CrossRef]

2. Siegel, R.L.; Miller, K.D.; Jemal, A. Cancer statistics, 2020. CA Cancer J. Clin. 2020, 70, 7-30. [CrossRef]

3. Bray, F.; Ferlay, J.; Soerjomataram, I.; Siegel, R.L.; Torre, L.A.; Jemal, A. Global cancer statistics 2018: GLOBOCAN estimates of incidence and mortality worldwide for 36 cancers in 185 countries. CA Cancer J. Clin. 2018, 68, 394-424. [CrossRef] [PubMed]

4. da Costa, W.L., Jr.; Oluyomi, A.O.; Thrift, A.P. Trends in the Incidence of Pancreatic Adenocarcinoma in All 50 United States Examined Through an Age-Period-Cohort Analysis. JNCI Cancer Spectr. 2020, 4, pkaa033. [CrossRef]

5. Rawla, P.; Sunkara, T.; Gaduputi, V. Epidemiology of Pancreatic Cancer: Global Trends, Etiology and Risk Factors. World J. Oncol. 2019, 10, 10-27. [CrossRef]

6. Lin, Q.J.; Yang, F.; Jin, C.; Fu, D.L. Current status and progress of pancreatic cancer in China. World J. Gastroenterol. 2015, 21, 7988-8003. [CrossRef]

7. Shen, G.Q.; Aleassa, E.M.; Walsh, R.M.; Morris-Stiff, G. Next-Generation Sequencing in Pancreatic Cancer. Pancreas 2019, 48, 739-748. [CrossRef] [PubMed]

8. Bortesi, L.; Pesci, A.; Bogina, G.; Castelli, P.; Zamboni, G. Ductal Adenocarcinoma of the Pancreas. Surg. Pathol. Clin. 2011, 4, 487-521. [CrossRef]

9. CancerResearchUK. Pancreatic Cancer Statistics 2015-2017. Available online: http://www.cancerresearchuk.org/healthprofessional/cancer-statistics/statistics-by-cancer-type/pancreatic-cancer (accessed on 15 July 2020).

10. Pancreatic Cancer UK. Pancreatic Cancer Statistics. Available online: https://www.pancreaticcancer.org.uk/what-we-do/mediacentre/pancreatic-cancer-statistics (accessed on 2 July 2020).

11. Siegel, R.L.; Miller, K.D.; Jemal, A. Cancer statistics, 2018. CA Cancer J. Clin. 2018, 68, 7-30. [CrossRef] [PubMed]

12. Huang, L.; Jansen, L.; Balavarca, Y.; Molina-Montes, E.; Babaei, M.; van der Geest, L.; Lemmens, V.; Van Eycken, L.; De Schutter, H.; Johannesen, T.B.; et al. Resection of pancreatic cancer in Europe and USA: An international large-scale study highlighting large variations. Gut 2019, 68, 130. [CrossRef]

13. Shimizu, Y.; Yasui, K.; Matsueda, K.; Yanagisawa, A.; Yamao, K. Small carcinoma of the pancreas is curable: New computed tomography finding, pathological study and postoperative results from a single institute. J. Gastroenterol. Hepatol. 2005, 20, 1591-1594. [CrossRef]

14. Ishikawa, O.; Ohigashi, H.; Imaoka, S.; Nakaizumi, A.; Uehara, H.; Kitamura, T.; Kuroda, C. Minute carcinoma of the pancreas measuring $1 \mathrm{~cm}$ or less in diameter-Collective review of Japanese case reports. Hepatogastroenterology 1999, 46, 8-15.

15. Yachida, S.; Jones, S.; Bozic, I.; Antal, T.; Leary, R.; Fu, B.; Kamiyama, M.; Hruban, R.H.; Eshleman, J.R.; Nowak, M.A.; et al. Distant metastasis occurs late during the genetic evolution of pancreatic cancer. Nature 2010, 467, 1114-1117. [CrossRef]

16. Ghaneh, P.; Costello, E.; Neoptolemos, J.P. Biology and management of pancreatic cancer. Gut 2007, 56, 1134-1152. [CrossRef] 
17. Brand, R.E.; Nolen, B.M.; Zeh, H.J.; Allen, P.J.; Eloubeidi, M.A.; Goldberg, M.; Elton, E.; Arnoletti, J.P.; Christein, J.D.; Vickers, S.M.; et al. Serum biomarker panels for the detection of pancreatic cancer. Clin. Cancer Res. 2011, 17, 805-816. [CrossRef]

18. Chan, A.; Diamandis, E.P.; Blasutig, I.M. Strategies for discovering novel pancreatic cancer biomarkers. J. Proteom. 2013, 81, 126-134. [CrossRef]

19. Tempero, M.A.; Uchida, E.; Takasaki, H.; Burnett, D.A.; Steplewski, Z.; Pour, P.M. Relationship of carbohydrate antigen 19-9 and Lewis antigens in pancreatic cancer. Cancer Res. 1987, 47, 5501-5503.

20. FDA. FDA 510K Summary. Available online: https://www.accessdata.fda.gov/cdrh_docs/pdf2/K020566.pdf (accessed on 20 February 2021).

21. Park, H.-D.; Kang, E.-S.; Kim, J.-W.; Lee, K.-T.; Lee, K.H.; Park, Y.S.; Park, J.-O.; Lee, J.; Heo, J.S.; Choi, S.H.; et al. Serum CA19-9, cathepsin D, and matrix metalloproteinase-7 as a diagnostic panel for pancreatic ductal adenocarcinoma. Proteomics 2012, 12, 3590-3597. [CrossRef] [PubMed]

22. Ritts, R.E.; Pitt, H.A. CA 19-9 in pancreatic cancer. Surg. Oncol. Clin. N. Am. 1998, 7, 93-101. [CrossRef]

23. Duffy, M.J.; van Dalen, A.; Haglund, C.; Hansson, L.; Klapdor, R.; Lamerz, R.; Nilsson, O.; Sturgeon, C.; Topolcan, O. Clinical utility of biochemical markers in colorectal cancer: European Group on Tumour Markers (EGTM) guidelines. Eur. J. Cancer 2003, 39, 718-727. [CrossRef]

24. Ballehaninna, U.K.; Chamberlain, R.S. The clinical utility of serum CA 19-9 in the diagnosis, prognosis and management of pancreatic adenocarcinoma: An evidence based appraisal. J. Gastrointest. Oncol. 2012, 3, 105-119. [CrossRef]

25. Passerini, R.; Cassatella, M.C.; Boveri, S.; Salvatici, M.; Radice, D.; Zorzino, L.; Galli, C.; Sandri, M.T. The pitfalls of CA19-9: Routine testing and comparison of two automated immunoassays in a reference oncology center. Am. J. Clin. Pathol. 2012, 138, 281-287. [CrossRef]

26. Duffy, M.J.; Sturgeon, C.; Lamerz, R.; Haglund, C.; Holubec, V.L.; Klapdor, R.; Nicolini, A.; Topolcan, O.; Heinemann, V. Tumor markers in pancreatic cancer: A European Group on Tumor Markers (EGTM) status report. Ann. Oncol. 2010, $21,441-447$. [CrossRef]

27. Herlyn, M.; Steplewski, Z.; Herlyn, D.; Koprowski, H. Colorectal carcinoma-specific antigen: Detection by means of monoclonal antibodies. Proc. Natl. Acad. Sci. USA 1979, 76, 1438-1442. [CrossRef] [PubMed]

28. Hotakainen, K.; Tanner, P.; Alfthan, H.; Haglund, C.; Stenman, U.H. Comparison of three immunoassays for CA 19-9. Clin. Chim. Acta 2009, 400, 123-127. [CrossRef] [PubMed]

29. Steinberg, W. The clinical utility of the CA 19-9 tumor-associated antigen. Am. J. Gastroenterol. 1990, 85, 350-355.

30. Stern, P.; Friedecky, B.; Bartos, V.; Bezdickova, D.; Vavrova, J.; Uhrova, J.; Rozprimova, L.; Zima, T.; Palicka, V. Comparison of different immunoassays for CA 19-9. Clin. Chem. Lab. Med. 2001, 39, 1278-1282. [CrossRef]

31. Goh, S.K.; Gold, G.; Christophi, C.; Muralidharan, V. Serum carbohydrate antigen 19-9 in pancreatic adenocarcinoma: A mini review for surgeons. ANZ J. Surg. 2017, 87, 987-992. [CrossRef]

32. Jain, K.K. The Handbook of Biomarkers; Humana Press: Totowa, NJ, USA, 2010.

33. Young, M.R.; Wagner, P.D.; Ghosh, S.; Rinaudo, J.A.; Baker, S.G.; Zaret, K.S.; Goggins, M.; Srivastava, S. Validation of Biomarkers for Early Detection of Pancreatic Cancer: Summary of The Alliance of Pancreatic Cancer Consortia for Biomarkers for Early Detection Workshop. Pancreas 2018, 47, 135-141. [CrossRef]

34. Moher, D.; Liberati, A.; Tetzlaff, J.; Altman, D.G. Preferred reporting items for systematic reviews and meta-analyses: The PRISMA statement. PloS Med. 2009, 6, e1000097. [CrossRef]

35. Whiting, P.F.; Rutjes, A.W.; Westwood, M.E.; Mallett, S.; Deeks, J.J.; Reitsma, J.B.; Leeflang, M.M.; Sterne, J.A.; Bossuyt, P.M. QUADAS-2: A revised tool for the quality assessment of diagnostic accuracy studies. Ann. Intern. Med. 2011, 155, 529-536. [CrossRef]

36. Whiting, P.; Rutjes, A.W.; Reitsma, J.B.; Bossuyt, P.M.; Kleijnen, J. The development of QUADAS: A tool for the quality assessment of studies of diagnostic accuracy included in systematic reviews. BMC Med. Res. Methodol. 2003, 3, 25. [CrossRef]

37. Gold, D.V.; Goggins, M.; Modrak, D.E.; Newsome, G.; Liu, M.; Shi, C.; Hruban, R.H.; Goldenberg, D.M. Detection of early-stage pancreatic adenocarcinoma. Cancer Epidemiol. Biomark. Prev. 2010, 19, 2786-2794. [CrossRef] [PubMed]

38. Joergensen, M.T.; Brunner, N.; De Muckadell, O.B.S. Comparison of Circulating MMP-9, TIMP-1 and CA19-9 in the Detection of Pancreatic Cancer. Anticancer Res. 2010, 30, 587-592. [PubMed]

39. Marten, A.; Buchler, M.W.; Werft, W.; Wente, M.N.; Kirschfink, M.; Schmidt, J. Soluble iC3b as an Early Marker for Pancreatic Adenocarcinoma Is Superior to CA19.9 and Radiology. J. Immunother. 2010, 33, 219-224. [CrossRef]

40. Capello, M.; Cappello, P.; Linty, F.C.; Chiarle, R.; Sperduti, I.; Novarino, A.; Salacone, P.; Mandili, G.; Naccarati, A.; Sacerdote, C.; et al. Autoantibodies to Ezrin are an early sign of pancreatic cancer in humans and in genetically engineered mouse models. $J$. Hematol. Oncol. 2013, 6, 67. [CrossRef] [PubMed]

41. Gold, D.V.; Gaedcke, J.; Ghadimi, B.M.; Goggins, M.; Hruban, R.H.; Liu, M.L.; Newsome, G.; Goldenberg, D.M. PAM4 enzyme immunoassay alone and in combination with CA 19-9 for the detection of pancreatic adenocarcinoma. Cancer 2013, 119, 522-528. [CrossRef]

42. Kobayashi, T.; Nishiumi, S.; Ikeda, A.; Yoshie, T.; Sakai, A.; Matsubara, A.; Izumi, Y.; Tsumura, H.; Tsuda, M.; Nishisaki, H.; et al. A novel serum metabolomics-based diagnostic approach to pancreatic cancer. Cancer Epidemiol. Biomark. Prev. 2013, 22, 571-579. [CrossRef] 
43. Li, A.; Yu, J.; Kim, H.; Wolfgang, C.L.; Canto, M.I.; Hruban, R.H.; Goggins, M. MicroRNA array analysis finds elevated serum miR-1290 accurately distinguishes patients with low-stage pancreatic cancer from healthy and disease controls. Clin. Cancer Res. 2013, 19, 3600-3610. [CrossRef]

44. Zhao, C.; Zhang, J.; Zhang, S.; Yu, D.; Chen, Y.; Liu, Q.; Shi, M.; Ni, C.; Zhu, M. Diagnostic and biological significance of microRNA-192 in pancreatic ductal adenocarcinoma. Oncol. Rep. 2013, 30, 276-284. [CrossRef] [PubMed]

45. Chung, H.W.; Lim, J.B. Clinical significance of elevated serum soluble CD40 ligand levels as a diagnostic and prognostic tumor marker for pancreatic ductal adenocarcinoma. J. Transl. Med. 2014, 12, 102. [CrossRef] [PubMed]

46. Lee, M.J.; Na, K.; Jeong, S.K.; Lim, J.S.; Kim, S.A.; Song, S.Y.; Kim, H.; Hancock, W.S.; Paik, Y.K. Identification of human complement factor B as a novel biomarker candidate for pancreatic ductal adenocarcinoma. J. Proteome Res. 2014, 13, 4878-4888. [CrossRef] [PubMed]

47. Nolen, B.M.; Brand, R.E.; Prosser, D.; Velikokhatnaya, L.; Allen, P.J.; Zeh, H.J.; Grizzle, W.E.; Huang, Y.; Lomakin, A.; Lokshin, A.E. Prediagnostic serum biomarkers as early detection tools for pancreatic cancer in a large prospective cohort study. PLoS ONE 2014, 9, e94928. [CrossRef] [PubMed]

48. Ren, C.; Chen, Y.; Han, C.; Fu, D.; Chen, H. Plasma interleukin-11 (IL-11) levels have diagnostic and prognostic roles in patients with pancreatic cancer. Tumour Biol. 2014, 35, 11467-11472. [CrossRef]

49. Schultz, N.A.; Dehlendorff, C.; Jensen, B.V.; Bjerregaard, J.K.; Nielsen, K.R.; Bojesen, S.E.; Calatayud, D.; Nielsen, S.E.; Yilmaz, M.; Holländer, N.H.; et al. MicroRNA biomarkers in whole blood for detection of pancreatic cancer. JAMA 2014, 311, 392-404. [CrossRef] [PubMed]

50. Yang, J.Y.; Sun, Y.W.; Liu, D.J.; Zhang, J.F.; Li, J.; Hua, R. MicroRNAs in stool samples as potential screening biomarkers for pancreatic ductal adenocarcinoma cancer. Am. J. Cancer Res. 2014, 4, 663-673. [PubMed]

51. Zhang, P.J.; Zou, M.; Wen, X.Y.; Gu, F.; Li, J.; Liu, G.X.; Dong, J.X.; Deng, X.X.; Gao, J.; Li, X.L.; et al. Development of serum parameters panels for the early detection of pancreatic cancer. Int. J. Cancer 2014, 134, 2646-2655. [CrossRef]

52. Debernardi, S.; Massat, N.J.; Radon, T.P.; Sangaralingam, A.; Banissi, A.; Ennis, D.P.; Dowe, T.; Chelala, C.; Pereira, S.P.; Kocher, H.M.; et al. Noninvasive urinary miRNA biomarkers for early detection of pancreatic adenocarcinoma. Am. J. Cancer Res. 2015, 5, 3455-3466.

53. Han, S.X.; Zhou, X.; Sui, X.; He, C.C.; Cai, M.J.; Ma, J.L.; Zhang, Y.Y.; Zhou, C.Y.; Ma, C.X.; Varela-Ramirez, A.; et al. Serum dickkopf-1 is a novel serological biomarker for the diagnosis and prognosis of pancreatic cancer. Oncotarget 2015, 6, 19907-19917. [CrossRef]

54. Melo, S.A.; Luecke, L.B.; Kahlert, C.; Fernandez, A.F.; Gammon, S.T.; Kaye, J.; LeBleu, V.S.; Mittendorf, E.A.; Weitz, J.; Rahbari, N.; et al. Glypican-1 identifies cancer exosomes and detects early pancreatic cancer. Nature 2015, 523, 177-182. [CrossRef]

55. Radon, T.P.; Massat, N.J.; Jones, R.; Alrawashdeh, W.; Dumartin, L.; Ennis, D.; Duffy, S.W.; Kocher, H.M.; Pereira, S.P.; Guarner, L.; et al. Identification of a Three-Biomarker Panel in Urine for Early Detection of Pancreatic Adenocarcinoma. Clin. Cancer Res. 2015, 21, 3512-3521. [CrossRef] [PubMed]

56. Ankeny, J.S.; Court, C.M.; Hou, S.; Li, Q.; Song, M.; Wu, D.; Chen, J.F.; Lee, T.; Lin, M.; Sho, S.; et al. Circulating tumour cells as a biomarker for diagnosis and staging in pancreatic cancer. Br. J. Cancer 2016, 114, 1367-1375. [CrossRef]

57. Guo, X.; Lv, X.; Fang, C.; Wang, F.; Wang, D.; Zhao, J.; Ma, Y.; Xue, Y.; Bai, Q.; Yao, X.; et al. Dysbindin as a novel biomarker for pancreatic ductal adenocarcinoma identified by proteomic profiling. Int. J. Cancer 2016, 139, 1821-1829. [CrossRef]

58. Henriksen, S.D.; Madsen, P.H.; Larsen, A.C.; Johansen, M.B.; Drewes, A.M.; Pedersen, I.S.; Krarup, H.; Thorlacius-Ussing, O. Cell-free DNA promoter hypermethylation in plasma as a diagnostic marker for pancreatic adenocarcinoma. Clin. Epigenetics 2016, 8, 1-12. [CrossRef]

59. Sogawa, K.; Takano, S.; Iida, F.; Satoh, M.; Tsuchida, S.; Kawashima, Y.; Yoshitomi, H.; Sanda, A.; Kodera, Y.; Takizawa, H.; et al. Identification of a novel serum biomarker for pancreatic cancer, C4b-binding protein $\alpha$-chain (C4BPA) by quantitative proteomic analysis using tandem mass tags. Br. J. Cancer 2016, 115, 949-956. [CrossRef]

60. Yoneyama, T.; Ohtsuki, S.; Honda, K.; Kobayashi, M.; Iwasaki, M.; Uchida, Y.; Okusaka, T.; Nakamori, S.; Shimahara, M.; Ueno, T.; et al. Identification of IGFBP2 and IGFBP3 As Compensatory Biomarkers for CA19-9 in Early-Stage Pancreatic Cancer Using a Combination of Antibody-Based and LC-MS/MS-Based Proteomics. PLoS ONE 2016, 11, e0161009. [CrossRef] [PubMed]

61. Balasenthil, S.; Huang, Y.; Liu, S.; Marsh, T.; Chen, J.; Stass, S.A.; KuKuruga, D.; Brand, R.; Chen, N.; Frazier, M.L.; et al. A Plasma Biomarker Panel to Identify Surgically Resectable Early-Stage Pancreatic Cancer. J. Natl. Cancer Inst. 2017, 109. [CrossRef] [PubMed]

62. Yang, K.S.; Im, H.; Hong, S.; Pergolini, I.; del Castillo, A.F.; Wang, R.; Clardy, S.; Huang, C.-H.; Pille, C.; Ferrone, S.; et al. Multiparametric plasma EV profiling facilitates diagnosis of pancreatic malignancy. Sci. Transl. Med. 2017, 9, eaal3226. [CrossRef] [PubMed]

63. Capello, M.; Bantis, L.; Scelo, G.; Zhao, Y.; Dhillon, D.; Wang, H.; Abbruzzese, J.; Maitra, A.; Tempero, M.; Brand, R.; et al. Sequential validation of bloodbased protein biomarker candidates for early-stage pancreatic cancer. In Proceedings of the 107th Annual Meeting of the American Association for Cancer Research, AACR 2016, New Orleans, LA, USA, 16-20 April 2016; Volume 76.

64. Hussein, N.A.; Kholy, Z.A.; Anwar, M.M.; Ahmad, M.A.; Ahmad, S.M. Plasma miR-22-3p, miR-642b-3p and miR-885-5p as diagnostic biomarkers for pancreatic cancer. J. Cancer Res. Clin. Oncol. 2017, 143, 83-93. [CrossRef] 
65. Kaur, S.; Smith, L.; Patel, A.; Menning, M.; Watley, D.; Malik, S.; Krishn, S.; Mallya, K.; Aithal, A.; Sasson, A.; et al. A Combination of MUC5AC and CA19-9 Improves the Diagnosis of Pancreatic Cancer: A Multicenter Study. Am. J. Gastroenterol. 2017, 112, 172-183. [CrossRef] [PubMed]

66. Kim, J.; Bamlet, W.R.; Oberg, A.L.; Chaffee, K.G.; Donahue, G.; Cao, X.J.; Chari, S.; Garcia, B.A.; Petersen, G.M.; Zaret, K.S. Detection of early pancreatic ductal adenocarcinoma with thrombospondin-2 and CA19-9 blood markers. Sci. Transl. Med. 2017, 9, eaah5583. [CrossRef] [PubMed]

67. Lai, X.Y.; Wang, M.; McElyea, S.D.; Sherman, S.; House, M.; Korc, M. A microRNA signature in circulating exosomes is superior to exosomal glypican-1 levels for diagnosing pancreatic cancer. Cancer Lett. 2017, 393, 86-93. [CrossRef]

68. Park, J.; Choi, Y.; Namkung, J.; Yi, S.G.; Kim, H.; Yu, J.; Kim, Y.; Kwon, M.S.; Kwon, W.; Oh, D.Y.; et al. Diagnostic performance enhancement of pancreatic cancer using proteomic multimarker panel. Oncotarget 2017, 8, 93117-93130. [CrossRef]

69. Schott, S.; Yang, R.X.; Stocker, S.; Canzian, F.; Giese, N.; Bugert, P.; Bergmann, F.; Strobel, O.; Hackert, T.; Sohn, C.; et al. HYAL2 methylation in peripheral blood as a potential marker for the detection of pancreatic cancer-a case control study. Oncotarget 2017, 8, 67614-67625. [CrossRef] [PubMed]

70. Arasaradnam, R.P.; Wicaksono, A.; O’Brien, H.; Kocher, H.M.; Covington, J.A.; Crnogorac-Jurcevic, T. Noninvasive Diagnosis of Pancreatic Cancer Through Detection of Volatile Organic Compounds in Urine. Gastroenterology 2018, 154, 485-487.e481. [CrossRef] [PubMed]

71. Dong, D.; Jia, L.; Zhang, L.; Ma, N.; Zhang, A.; Zhou, Y.; Ren, L. Periostin and CA242 as potential diagnostic serum biomarkers complementing CA19.9 in detecting pancreatic cancer. Cancer Sci. 2018, 109, 2841-2851. [CrossRef]

72. Guo, X.B.; Yin, H.S.; Wang, J.Y. Evaluating the diagnostic and prognostic value of long non-coding RNA SNHG15 in pancreatic ductal adenocarcinoma. Eur. Rev. Med. Pharm. Sci. 2018, 22, 5892-5898. [CrossRef]

73. Lewis, J.M.; Vyas, A.D.; Qiu, Y.; Messer, K.S.; White, R.; Heller, M.J. Integrated Analysis of Exosomal Protein Biomarkers on Alternating Current Electrokinetic Chips Enables Rapid Detection of Pancreatic Cancer in Patient Blood. ACS Nano 2018, 12, 3311-3320. [CrossRef]

74. Mellby, L.D.; Nyberg, A.P.; Johansen, J.S.; Wingren, C.; Nordestgaard, B.G.; Bojesen, S.E.; Mitchell, B.L.; Sheppard, B.C.; Sears, R.C.; Borrebaeck, C.A.K. Serum Biomarker Signature-Based Liquid Biopsy for Diagnosis of Early-Stage Pancreatic Cancer. J. Clin. Oncol. 2018, 36, 2887-2894. [CrossRef] [PubMed]

75. Michael Traeger, M.; Rehkaemper, J.; Ullerich, H.; Steinestel, K.; Wardelmann, E.; Senninger, N.; Abdallah Dhayat, S. The ambiguous role of microRNA-205 and its clinical potential in pancreatic ductal adenocarcinoma. J. Cancer Res. Clin. Oncol. 2018, 144, 2419-2431. [CrossRef]

76. Zhou, C.Y.; Dong, Y.P.; Sun, X.; Sui, X.; Zhu, H.; Zhao, Y.Q.; Zhang, Y.Y.; Mason, C.; Zhu, Q.; Han, S.X. High levels of serum glypican-1 indicate poor prognosis in pancreatic ductal adenocarcinoma. Cancer Med. 2018, 7, 5525-5533. [CrossRef]

77. Berger, A.W.; Schwerdel, D.; Reinacher-Schick, A.; Uhl, W.; Algül, H.; Friess, H.; Janssen, K.-P.; König, A.; Ghadimi, M.; Gallmeier, E.; et al. A Blood-Based Multi Marker Assay Supports the Differential Diagnosis of Early-Stage Pancreatic Cancer. Theranostics 2019, 9, 1280-1287. [CrossRef]

78. Eissa, M.A.L.; Lerner, L.; Abdelfatah, E.; Shankar, N.; Canner, J.K.; Hasan, N.M.; Yaghoobi, V.; Huang, B.; Kerner, Z.; Takaesu, F.; et al. Promoter methylation of ADAMTS1 and BNC1 as potential biomarkers for early detection of pancreatic cancer in blood. Clin. Epigenetics 2019, 11, 59. [CrossRef] [PubMed]

79. Fahrmann, J.F.; Bantis, L.E.; Capello, M.; Scelo, G.; Dennison, J.B.; Patel, N.; Murage, E.; Vykoukal, J.; Kundnani, D.L.; Foretova, L.; et al. A Plasma-Derived Protein-Metabolite Multiplexed Panel for Early-Stage Pancreatic Cancer. J. Natl. Cancer Inst. 2019, 111, 372-379. [CrossRef]

80. Yu, S.; Li, Y.; Liao, Z.; Wang, Z.; Wang, Z.; Li, Y.; Qian, L.; Zhao, J.; Zong, H.; Kang, B.; et al. Plasma extracellular vesicle long RNA profiling identifies a diagnostic signature for the detection of pancreatic ductal adenocarcinoma. Gut 2020, 69, 540-550. [CrossRef]

81. Takahashi, K.; Ota, Y.; Kogure, T.; Suzuki, Y.; Iwamoto, H.; Yamakita, K.; Kitano, Y.; Fujii, S.; Haneda, M.; Patel, T.; et al. Circulating extracellular vesicle-encapsulated HULC is a potential biomarker for human pancreatic cancer. Cancer Sci. 2020, 111, 98-111. [CrossRef]

82. Wei, T.; Zhang, X.; Zhang, Q.; Yang, J.; Chen, Q.; Wang, J.; Li, X.; Chen, J.; Ma, T.; Li, G.; et al. Vimentin-positive circulating tumor cells as a biomarker for diagnosis and treatment monitoring in patients with pancreatic cancer. Cancer Lett. 2019, 452, 237-243. [CrossRef] [PubMed]

83. Yang, Z.; LaRiviere, M.J.; Ko, J.; Till, J.E.; Christensen, T.; Yee, S.S.; Black, T.A.; Tien, K.; Lin, A.; Shen, H.; et al. A Multianalyte Panel Consisting of Extracellular Vesicle miRNAs and mRNAs, cfDNA, and CA19-9 Shows Utility for Diagnosis and Staging of Pancreatic Ductal Adenocarcinoma. Clin. Cancer Res. 2020, 26, 3248-3258. [CrossRef] [PubMed]

84. Pang, Y.; Holmes, M.V.; Chen, Z.; Kartsonaki, C. A review of lifestyle, metabolic risk factors, and blood-based biomarkers for early diagnosis of pancreatic ductal adenocarcinoma. J. Gastroenterol. Hepatol. 2019, 34, 330-345. [CrossRef] [PubMed]

85. Becker, A.E.; Hernandez, Y.G.; Frucht, H.; Lucas, A.L. Pancreatic ductal adenocarcinoma: Risk factors, screening, and early detection. World J. Gastroenterol. 2014, 20, 11182-11198. [CrossRef]

86. Ghatnekar, O.; Andersson, R.; Svensson, M.; Persson, U.; Ringdahl, U.; Zeilon, P.; Borrebaeck, C.A. Modelling the benefits of early diagnosis of pancreatic cancer using a biomarker signature. Int. J. Cancer 2013, 133, 2392-2397. [CrossRef] [PubMed] 
87. Frampton, A.E.; Prado, M.M.; López-Jiménez, E.; Fajardo-Puerta, A.B.; Jawad, Z.A.R.; Lawton, P.; Giovannetti, E.; Habib, N.A.; Castellano, L.; Stebbing, J.; et al. Glypican-1 is enriched in circulating-exosomes in pancreatic cancer and correlates with tumor burden. Oncotarget 2018, 9, 19006-19013. [CrossRef] [PubMed]

88. Zhang, W.H.; Wang, W.Q.; Han, X.; Gao, H.L.; Li, T.J.; Xu, S.S.; Li, S.; Xu, H.X.; Li, H.; Ye, L.Y.; et al. Advances on diagnostic biomarkers of pancreatic ductal adenocarcinoma: A systems biology perspective. Comput. Struct. Biotechnol. J. 2020, 18, 3606-3614. [CrossRef]

89. Almeida, P.P.; Cardoso, C.P.; de Freitas, L.M. PDAC-ANN: An artificial neural network to predict pancreatic ductal adenocarcinoma based on gene expression. BMC Cancer 2020, 20, 82. [CrossRef]

90. Muhammad, W.; Hart, G.R.; Nartowt, B.; Farrell, J.J.; Johung, K.; Liang, Y.; Deng, J. Pancreatic Cancer Prediction Through an Artificial Neural Network. Front. Artif. Intell. 2019, 2, 2. [CrossRef] [PubMed]

91. Marya, N.B.; Powers, P.D.; Chari, S.T.; Gleeson, F.C.; Leggett, C.L.; Abu Dayyeh, B.K.; Chandrasekhara, V.; Iyer, P.G.; Majumder, S.; Pearson, R.K.; et al. Utilisation of artificial intelligence for the development of an EUS-convolutional neural network model trained to enhance the diagnosis of autoimmune pancreatitis. Gut 2020. [CrossRef] [PubMed]

92. Khatri, I.; Bhasin, M.K. A Transcriptomics-Based Meta-Analysis Combined With Machine Learning Identifies a Secretory Biomarker Panel for Diagnosis of Pancreatic Adenocarcinoma. Front. Genet. 2020, 11, 572284. [CrossRef] [PubMed]

93. Chung, W.Y.; Correa, E.; Yoshimura, K.; Chang, M.C.; Dennison, A.; Takeda, S.; Chang, Y.T. Using probe electrospray ionization mass spectrometry and machine learning for detecting pancreatic cancer with high performance. Am. J. Transl. Res. 2020, 12, 171-179. [PubMed]

94. Sollie, S.; Michaud, D.S.; Sarker, D.; Karagiannis, S.N.; Josephs, D.H.; Hammar, N.; Santaolalla, A.; Walldius, G.; Garmo, H.; Holmberg, L.; et al. Chronic inflammation markers are associated with risk of pancreatic cancer in the Swedish AMORIS cohort study. BMC Cancer 2019, 19, 858. [CrossRef]

95. de la Fuente, J.; Sharma, A.; Chari, S.; Majumder, S. Peripheral blood monocyte counts are elevated in the pre-diagnostic phase of pancreatic cancer: A population based study. Pancreatology 2019, 19, 1043-1048. [CrossRef]

96. Cui, Y.; Shu, X.O.; Li, H.L.; Yang, G.; Wen, W.; Gao, Y.T.; Cai, Q.; Rothman, N.; Yin, H.Y.; Lan, Q.; et al. Prospective study of urinary prostaglandin E2 metabolite and pancreatic cancer risk. Int. J. Cancer 2017, 141, 2423-2429. [CrossRef] [PubMed]

97. Jenkinson, C.; Elliott, V.L.; Evans, A.; Oldfield, L.; Jenkins, R.E.; O’Brien, D.P.; Apostolidou, S.; Gentry-Maharaj, A.; Fourkala, E.O.; Jacobs, I.J.; et al. Decreased Serum Thrombospondin-1 Levels in Pancreatic Cancer Patients Up to 24 Months Prior to Clinical Diagnosis: Association with Diabetes Mellitus. Clin. Cancer Res. 2016, 22, 1734-1743. [CrossRef] [PubMed]

98. O'Brien, D.P.; Sandanayake, N.S.; Jenkinson, C.; Gentry-Maharaj, A.; Apostolidou, S.; Fourkala, E.O.; Camuzeaux, S.; Blyuss, O.; Gunu, R.; Dawnay, A.; et al. Serum CA19-9 is significantly upregulated up to 2 years before diagnosis with pancreatic cancer: Implications for early disease detection. Clin. Cancer Res. 2015, 21, 622-631. [CrossRef]

99. Capello, M.; Bantis, L.E.; Scelo, G.; Zhao, Y.; Li, P.; Dhillon, D.S.; Patel, N.J.; Kundnani, D.L.; Wang, H.; Abbruzzese, J.L.; et al. Sequential Validation of Blood-Based Protein Biomarker Candidates for Early-Stage Pancreatic Cancer. J. Natl. Cancer Inst. 2017, 109, djw266. [CrossRef] [PubMed]

100. Mirus, J.E.; Zhang, Y.; Li, C.I.; Lokshin, A.E.; Prentice, R.L.; Hingorani, S.R.; Lampe, P.D. Cross-species antibody microarray interrogation identifies a 3-protein panel of plasma biomarkers for early diagnosis of pancreas cancer. Clin. Cancer Res. 2015, 21, 1764-1771. [CrossRef] [PubMed]

101. Magis, A.T.; Rappaport, N.; Conomos, M.P.; Omenn, G.S.; Lovejoy, J.C.; Hood, L.; Price, N.D. Untargeted longitudinal analysis of a wellness cohort identifies markers of metastatic cancer years prior to diagnosis. Sci. Rep. 2020, 10, 16275. [CrossRef]

102. Moore, H.M.; Kelly, A.B.; Jewell, S.D.; McShane, L.M.; Clark, D.P.; Greenspan, R.; Hayes, D.F.; Hainaut, P.; Kim, P.; Mansfield, E.A.; et al. Biospecimen reporting for improved study quality (BRISQ). Cancer Cytopathol. 2011, 119, 92-101. [CrossRef] [PubMed]

103. Cohen, J.F.; Korevaar, D.A.; Altman, D.G.; Bruns, D.E.; Gatsonis, C.A.; Hooft, L.; Irwig, L.; Levine, D.; Reitsma, J.B.; de Vet, H.C.W.; et al. STARD 2015 guidelines for reporting diagnostic accuracy studies: Explanation and elaboration. BMJ Open 2016, 6, e012799. [CrossRef]

104. Altman, D.G.; McShane, L.M.; Sauerbrei, W.; Taube, S.E. Reporting Recommendations for Tumor Marker Prognostic Studies (REMARK): Explanation and elaboration. PLoS Med. 2012, 9, e1001216. [CrossRef]

105. Schulz, K.F.; Altman, D.G.; Moher, D. CONSORT 2010 statement: Updated guidelines for reporting parallel group randomised trials. BMJ 2010, 340, c332. [CrossRef]

106. Crosby, D.; Lyons, N.; Greenwood, E.; Harrison, S.; Hiom, S.; Moffat, J.; Quallo, T.; Samuel, E.; Walker, I. A roadmap for the early detection and diagnosis of cancer. Lancet Oncol. 2020, 21, 1397-1399. [CrossRef]

107. Kunovsky, L.; Tesarikova, P.; Kala, Z.; Kroupa, R.; Kysela, P.; Dolina, J.; Trna, J. The Use of Biomarkers in Early Diagnostics of Pancreatic Cancer. Can. J. Gastroenterol. Hepatol. 2018, 2018, 5389820. [CrossRef] [PubMed]

108. Paulovich, A.G.; Whiteaker, J.R.; Hoofnagle, A.N.; Wang, P. The interface between biomarker discovery and clinical validation: The tar pit of the protein biomarker pipeline. Proteom. Clin. Appl. 2008, 2, 1386-1402. [CrossRef]

109. Rifai, N.; Gillette, M.A.; Carr, S.A. Protein biomarker discovery and validation: The long and uncertain path to clinical utility. Nat. Biotechnol. 2006, 24, 971-983. [CrossRef] [PubMed]

110. Root, A.; Allen, P.; Tempst, P.; Yu, K. Protein Biomarkers for Early Detection of Pancreatic Ductal Adenocarcinoma: Progress and Challenges. Cancers 2018, 10, 67. [CrossRef] [PubMed]

111. Immunovia. Available online: https:/ /immunovia.com/about-us (accessed on 25 August 2020). 
112. Debernardi, S.; O’Brien, H.; Algahmdi, A.S.; Malats, N.; Stewart, G.D.; Plješa-Ercegovac, M.; Costello, E.; Greenhalf, W.; Saad, A.; Roberts, R.; et al. A combination of urinary biomarker panel and PancRISK score for earlier detection of pancreatic cancer: A case-control study. PLoS Med. 2020, 17, e1003489. [CrossRef] [PubMed]

113. Blyuss, O.; Zaikin, A.; Cherepanova, V.; Munblit, D.; Kiseleva, E.M.; Prytomanova, O.M.; Duffy, S.W.; Crnogorac-Jurcevic, T. Development of PancRISK, a urine biomarker-based risk score for stratified screening of pancreatic cancer patients. Br. J. Cancer 2020, 122, 692-696. [CrossRef]

114. Clinicaltrials.gov. Early Detection of Pancreatic Adenocarcinoma (PDAC) Using a Panel of Biomarkers (UroPanc). Available online: https:/ / clinicaltrials.gov / ct2/show / NCT04449406 (accessed on 3 March 2021).

115. Cohen, J.D.; Li, L.; Wang, Y.; Thoburn, C.; Afsari, B.; Danilova, L.; Douville, C.; Javed, A.A.; Wong, F.; Mattox, A.; et al. Detection and localization of surgically resectable cancers with a multi-analyte blood test. Science 2018, 359, 926-930. [CrossRef]

116. Liu, M.C.; Oxnard, G.R.; Klein, E.A.; Swanton, C.; Seiden, M.V.; Consortium, C. Sensitive and specific multi-cancer detection and localization using methylation signatures in cell-free DNA. Ann. Oncol. 2020, 31, 745-759. [CrossRef]

117. Killock, D. CancerSEEK and destroy-A blood test for early cancer detection. Nat. Rev. Clin. Oncol. 2018, 15, 133. [CrossRef] 